\title{
Integrable GL(2) geometry and hydrodynamic partial differential equations
}

\author{
Abraham D. Smith
}

This article is a local analysis of integrable GL(2)-structures of degree 4. A GL(2)-structure of degree $n$ corresponds to a distribution of rational normal cones over a manifold of dimension $n+1$. Integrability corresponds to the existence of many submanifolds that are spanned by lines in the cones. These GL(2)-structures are important because they naturally arise from a certain family of second-order hyperbolic partial differential equations (PDEs) in three variables that are integrable via hydrodynamic reduction. Familiar examples include the wave equation, the first flow of the dKP hierarchy and the Boyer-Finley equation.

The main results are a structure theorem for integrable GL(2)-structures, a classification for connected integrable GL(2)structures and an equivalence between local integrable GL(2)-structures and Hessian hydrodynamic hyperbolic PDEs in three variables.

This yields natural geometric characterizations of the wave equation, the first flow of the dKP hierarchy and several others. It also provides an intrinsic, coordinate-free infrastructure to describe a large class of hydrodynamic integrable systems in three variables.

0 Introduction

$\begin{array}{lll}1 & \text { Background } & 747\end{array}$

1.1 Hessian hydrodynamic hyperbolic PDEs 749

$\begin{array}{lll}2 & \text { A preferred connection } & 754\end{array}$

$\begin{array}{lll}3 & 2-I n t e g r a b i l i t y & 759\end{array}$

$\begin{array}{lll}4 & 3 \text {-Integrability } & 762\end{array}$

$\begin{array}{lll}5 & \text { The classification } & 768\end{array}$ 
5.1 Symmetry reduction

6 GL(2) PDEs

6.1 Hyperbolic planar PDEs

7 Concluding remarks

782

Appendix A: The matrix $J(T)$

784

Acknowledgments

786

References

786

\section{Introduction}

A fundamental problem in analysis is to understand why some differential equations (particularly hyperbolic equations arising from wave-like equations or from differential geometry) are integrable. This problem is compounded by many competing notions of integrability [35]. Informally, a partial differential equation (PDE) is called integrable if it can be solved thanks to the existence of an infinite hierarchy of conservation laws or of a family of invariant foliations along characteristics. Integrable PDEs of all types are well studied in two $(1+1)$ independent variables; however, examples are increasingly rare and poorly understood in higher dimensions.

A particularly interesting class of integrable PDEs are those that can be integrated by decomposing the equation to a set of coupled first-order equations that represent traveling waves [31]. This technique is called "integration by hydrodynamic reduction," and it has been extensively studied for various special classes of second-order PDEs in three or more independent variables $[7,14-17,32,33]$.

Consider a second-order hyperbolic equation of the form $F\left(\xi^{i}, u, u_{i}, u_{i j}\right)=0$ on scalar functions $u\left(\xi^{1}, \xi^{2}, \xi^{3}\right)$. In the method of hydrodynamic reduction, one hopes to integrate the $\mathrm{PDE} F=0$ by stipulating that the solution function $u$ (and its derivatives) may be written as $u\left(R^{1}, \ldots, R^{k}\right)$ for an a priori unknown number $k$ of functions $R^{1}(\xi), \ldots$, $R^{k}(\xi)$ whose derivatives admit the "commuting" relations

$$
\frac{\partial}{\partial \xi^{2}} R^{i}=\rho_{2}^{i}(R) \frac{\partial}{\partial \xi^{1}} R^{i}, \quad \frac{\partial}{\partial \xi^{3}} R^{i}=\rho_{3}^{i}(R) \frac{\partial}{\partial \xi^{1}} R^{i}
$$


which also imply

$$
\frac{1}{\rho_{2}^{i}-\rho_{2}^{j}} \frac{\partial \rho_{2}^{i}}{\partial R^{j}}=\frac{1}{\rho_{3}^{i}-\rho_{3}^{j}} \frac{\partial \rho_{3}^{i}}{\partial R^{j}}, \quad \forall i \neq j .
$$

Equations (0.1) and (0.2) can be solved by other methods [31]. The reduction of $F=0$ to Equation (0.2) is called a $k$-parameter hydrodynamic reduction. The equation $F=0$ is called integrable via $k$-parameter hydrodynamic reduction if it admits an infinite family of $k$-parameter hydrodynamic reductions and this family is itself parametrized by $k$ functions of one variable. The functions $R^{i}\left(\xi^{1}, \xi^{2}, \xi^{3}\right)$ are called Riemann invariants, and their level sets define the foliations along characteristics in the informal definition above. See the works cited above, particularly [14, 15, 17], for examples and detailed exposition of this technique.

Many examples of these so-called hydrodynamic equations are known, such as the wave equation $u_{22}=u_{13}$, the dispersionless KadomtsevPetviashvili $(\mathrm{dKP})$ equation $u_{22}=\left(u_{1}-u u_{3}\right)_{3}$, the first flow of the dKP hierarchy $u_{22}=u_{13}-\frac{1}{2}\left(u_{33}\right)^{2}$, the Boyer-Finley equation $u_{22}+u_{33}=e^{u_{11}}$ and some well-known integrable hierarchies. Tests for hydrodynamic integrability also exist, but so far there is no intrinsic, coordinate-free theory that classifies and generates all of these equations.

For the case of second-order hydrodynamic equations in three variables that involve only the Hessian of the solution function, recent work by Ferapontov et al. reveals a relationship between the integrability of the PDEs, the symplectic contact symmetries of the equations and the equations' underlying degree-4 GL(2)-structures [14]. Integrable GL(2)structures are not well known in the literature; they arise in the search for exotic affine holonomies in differential geometry [5] and a similar conformal geometry appears useful in the equivalence problem for ordinary differential equations [24], but the application to PDEs is very recent [14, 27].

The purpose of this article is to fully analyze the local geometry of integrable GL(2)-structures of degree 4, with an eye towards understanding PDEs that are integrable via hydrodynamic reduction. The main result is a natural classification of local integrable GL(2)-structures and the associated PDEs. This is achieved using geometry $\grave{a}$ la Cartan, namely exterior differential systems, moving frames and the method of equivalence.

Here is a summary of the contents of this article, including abbreviated and non-technical versions of the main theorems:

Section 1 defines GL(2)-structures and their 2-integrability and 3 -integrability. The property of $k$-integrability involves the existence of 
"many" foliations by certain submanifolds of dimension $k$. Section 1 also introduces the motivating observation by Ferapontov et al. that hyperbolic PDEs in three variables that are integrable by means of hydrodynamic reductions lead to GL(2)-structures that are both 2-integrable and 3-integrable (abbreviated 2,3-integrable).

Section 2 contains a description of the necessary SL(2) representation theory and an explicit solution to the first-order equivalence problem for GL(2)-structures. Corollary 2.1 defines a global preferred connection for GL(2)-structures that has essential torsion in the direct sum of $\mathbb{R}^{3}, \mathbb{R}^{7}$, $\mathbb{R}^{9}$, and $\mathbb{R}^{11}$, each of which is an irreducible $\mathrm{SL}(2)$ module.

In Section 3, the exterior differential system describing 2-integrability for a GL(2)-structure is analyzed. The most important object in this article, the irreducible $\mathbb{R}^{9}$-valued torsion, is first emphasized in Theorem 3.1.

Theorem 3.1. A GL(2)-structure is 2-integrable if and only if its torsion takes values only in $\mathbb{R}^{9}$.

Section 4 provides Theorem 4.2, a complete local description of GL(2)structures that are 2,3-integrable. The immediate consequence of this theorem is that the value of the torsion at a single point completely determines the local structure of a 2,3-integrable GL(2)-structure.

Theorem 4.2. A GL(2)-structure is 2,3-integrable if and only if its structure equations are of the form

$$
\begin{aligned}
d \omega & =-\theta \wedge \omega+T(\omega \wedge \omega) \\
d \theta & =-\theta \wedge \theta+T^{2}(\omega \wedge \omega) \\
d T & =J(T)(\omega, \theta)
\end{aligned}
$$

for an essential torsion $T$ in $\mathbb{R}^{9}$ and a $9 \times 9$ matrix $J(T)$ depending on $T$, as in Appendix A.

Section 5 presents a classification of connected 2,3-integrable GL(2)structures. This classification is provided by a natural stratification of $\mathbb{R}^{9}$ into equivalence classes that are defined by the matrix $J(T)$. These equivalence classes are explicitly identified in Theorem 5.1.

Theorem 5.1. Every connected 2,3-integrable GL(2)-structure belongs to exactly one of 55 equivalence classes, which are given by a stratification of $\mathbb{R}^{9}$ into the root factorization types of real binary octic polynomials. 
In Section 6, the structure equations from Section 4 are applied to reproduce the integrable PDEs, yielding Theorem 6.1.

Theorem 6.1. Every local 2,3-integrable GL(2)-structure arises from a Hessian hydrodynamic hyperbolic PDE.

Therefore, the classification in Theorem 5.1 is also a classification of Hessian hydrodynamic hyperbolic PDEs. Some previously known examples of such PDEs are identified in the new classification, and several new integrable PDEs are constructed. Also, a relationship between Hessian hydrodynamic hyperbolic PDEs in three variables and hyperbolic PDEs in two variables (Monge-Ampère, Goursat, or generic type) is observed.

This work would have been impossible to complete without the uncanny speed and accuracy of computer algebra software, and there is no sense in retyping dozens of pages of formulas and identities. Thus, a reader looking for explicit computational details may be disappointed by this article (in which case I direct the reader to [27]). However, most proofs rely only on basic concepts from differential geometry, linear algebra and representation theory, and a reader comfortable with the standard methods of exterior differential systems (and their various software implementations) can readily supply the computational details. A MAPLE file containing the structure equations appearing in Theorem 4.2 and the Maurer-Cartan form appearing in Theorem 6.1 will remain available at [26] as long as possible. This file should also be bundled with this article on arXiv.org. The content of this file allows rapid reproduction of all results following Theorem 4.2.

Please note that the terms "hyperbolic" and "integrable" are heavily used in slightly different contexts throughout this article. "Hyperbolic" refers to hyperplanes having maximal intersection with certain projective varieties (for example, in Lemma 1.2), to PDEs in both two and three independent variables with appropriate leading symbol (for example, the wave equation) and to exterior differential systems with appropriate tableaux (for example, in the proof of Theorem 3.1). The term "integrable" refers both to PDEs that admit infinitely many exact solutions and to GL(2)-structures that admit many secant submanifolds (submanifolds that one might be tempted to also call "hyperbolic"). Of course, all of these concepts are intimately related, so the standard overloading of these definitions is reasonable.

\section{Background}

Let $\mathcal{V}_{n}$ denote the vector space of degree $n$ homogeneous polynomials in $x$ and $y$ with real coefficients. Identify $\mathcal{V}_{n}$ with $\mathbb{R}^{n+1}$ using the terms from the 
binomial theorem to produce a basis; for example, $\mathcal{V}_{4} \rightarrow \mathbb{R}^{5}$ by

$v_{-4} x^{4}+v_{-2} 4 x^{3} y+v_{0} 6 x^{2} y^{2}+v_{2} 4 x y^{3}+v_{4} y^{4} \mapsto\left(v_{-4}, v_{-2}, v_{0}, v_{2}, v_{4}\right) \in \mathbb{R}^{5}$.

Let $M$ denote a 5 -dimensional smooth manifold, and let $\mathcal{F}$ denote the $\mathcal{V}_{4^{-}}$ valued coframe bundle over $M$, so the fiber $\mathcal{F}_{p}$ is the set of all isomorphisms $\mathbf{T}_{p} M \rightarrow \mathcal{V}_{4}$. The coframe bundle $\mathcal{F}$ is a principal right $\operatorname{GL}\left(\mathcal{V}_{4}\right)$ bundle.

Recall that $\mathcal{V}_{n}$ is the unique irreducible representation of $\mathfrak{s l}(2)$ of dimension $n+1$. The action of $\mathfrak{s l}(2)$ on $\mathcal{V}_{n}$ is generated by

$$
X=y \frac{\partial}{\partial x}, \quad Y=-x \frac{\partial}{\partial y} \quad \text { and } \quad H=x \frac{\partial}{\partial x}-y \frac{\partial}{\partial y}
$$

Definition 1.1. A GL(2)-structure $\mathcal{B} \rightarrow M$ is a reduction of $\mathcal{F}$ with fiber group $\mathrm{GL}(2) \subset \mathrm{GL}\left(\mathcal{V}_{n}\right)$ infinitesimally generated by $X, Y, H$, and the scaling action $I$.

Note that throughout this article, the symbols GL(2) and $\mathfrak{g l}(2)$ are used to denote the abstract Lie group and its Lie algebra as well as any particular irreducible representations $\mathrm{GL}(2) \subset \mathrm{GL}\left(\mathcal{V}_{n}\right)$ and $\mathfrak{g l}(2) \subset \mathfrak{g l}\left(\mathcal{V}_{n}\right)$. Since $\mathrm{SL}(2)$ has a unique irreducible representation $\mathcal{V}_{n}$ in each dimension $n+1$, it is always clear from context which representation of GL(2) is being considered.

Generally, a GL(2)-structure is said to have "degree $n$ " if the base space $M$ has dimension $n+1$. The degree 3 case is thoroughly studied in [5], and various observations are made for all $n$ in [27]. This article considers only the degree 4 case. The PGL(2) action generated by $X, Y$ and $H$ is the symmetry group of a rational normal curve in $\mathbb{P}\left(\mathcal{V}_{4}\right)$. A rational normal curve is a curve of degree $n$ in $\mathbb{P}\left(\mathbb{R}^{n+1}\right)$. All such curves are $\operatorname{PGL}(n+1)$-equivalent to $\left\{\left[g^{n}: g^{n-1} h: \cdots: g h^{n-1}: g^{n}\right]\right\}[20]$. The de-projectivization of the rational normal curve is the rational normal cone, which has a GL(2) symmetry group and is usually described as $\left\{\left(g^{n}, g^{n-1} h, \ldots, g h^{n-1}, h^{n}\right)\right\} \subset \mathbb{R}^{n+1}$. Thus, the geometric content of Definition 1.1 is contained in the following lemma.

Lemma 1.1. A GL(2)-structure $\mathcal{B} \rightarrow M^{5}$ is equivalent to a distribution of rational normal cones $\mathbf{C} \subset \mathbf{T} M$. For any $b \in \mathcal{B}_{p}, b\left(\mathbf{C}_{p}\right)=\left\{(g x+h y)^{4}\right.$ : $g, h \in \mathbb{R}\} \subset \mathcal{V}_{4}$.

Definition 1.2 (Integrability). Let $\mathcal{B} \rightarrow M$ be a $\mathrm{GL}(2)$-structure. A $k$-dimensional linear subspace $E \subset \mathbf{T}_{p} M$ is $k$-secant if $E \cap \mathbf{C}_{p}$ is $k$ distinct lines. Equivalently, $E$ is $k$-secant if $E$ is spanned by $k$ vectors in $\mathbf{C}_{p}$. A 
$k$-dimensional submanifold $N \subset M$ is $k$-secant if $\mathbf{T}_{p} N$ is a k-secant subspace of $\mathbf{T}_{p} M$ for every $p \in N$. A GL(2)-structure is $k$-integrable if, for every $k$-secant linear subspace $E \in \mathrm{Gr}_{\mathrm{k}}(\mathbf{T} M)$, there exists a $k$-secant submanifold $N$ with $E=\mathbf{T}_{p} N$.

Locally, 1-integrability is uninteresting, as it is simply the existence of a local flow for a vector field. Since $M$ has dimension 5, being 4-secant is an open condition on $E \in \mathrm{Gr}_{4}(\mathbf{T} M)$; however, the condition is closed for $k=2$ (bi-secant) and $k=3$ (tri-secant). A GL(2)-structure that is both 2-integrable and 3-integrable is called "2,3-integrable," or simply "integrable."

Notice that all the definitions are projectively invariant. Indeed, one could equivalently study the principal right PGL(2) bundle over $M$ defined by the symmetries of rational normal curves in $P \mathbf{T} M$. When considering the de-projectivized GL(2) geometry, one occasionally encounters an additional $\mathbb{Z} / 2 \mathbb{Z}$ symmetry by $\pm I$. For the purposes of this article, it is easier to proceed using affine geometry and deal with this symmetry when it arises.

The geometric content of this article is a complete local description and classification of GL(2)-structures of degree 4 that are 2,3-integrable. Such GL(2)-structures are particularly interesting because of their deep connection to integrable PDEs. The remainder of this section outlines how Ferapontov, Hadjikos and Khusnutdinova obtain 2,3-integrable GL(2)-structures from certain hydrodynamic PDEs in [14].

\subsection{Hessian hydrodynamic hyperbolic PDEs}

The space of 1 -jets over $\mathbb{R}^{3} \rightarrow \mathbb{R}$ is $\mathbb{J}^{1}=\left\{\left(\xi^{i}, z, p_{i}\right)\right\}$ where $1 \leq i, j \leq 3$. The space of 2 -jets is $\mathbb{J}^{2}=\left\{\left(\xi^{j}, z, p_{i}, U_{i j}\right)\right\}$ where $1 \leq i, j \leq 3$ and $U_{i j}=U_{j i}$. The contact system on $\mathbb{J}^{2}$ is the differential ideal $\mathcal{J}^{2}$ generated by

$$
\left\{d z-p_{i} d \xi^{i}, d p_{i}-U_{i j} d \xi^{j}\right\}
$$

A second-order PDE on $u: \mathbb{R}^{3} \rightarrow \mathbb{R}$ is equivalent to the level set $F^{-1}(0)$ of some smooth function $F: \mathbb{J}^{2} \rightarrow \mathbb{R}$ on which the projection $\mathbb{J}^{2} \rightarrow \mathbb{J}^{1}$ is a submersion, and a solution is a function $u: \mathbb{R}^{3} \rightarrow \mathbb{R}$ whose jet-graph is a subset of $F^{-1}(0)$.

Recall that a section of $\mathbb{J}^{2}$ is the jet-graph of a function if and only if it is an integral of the contact system. A contact transformation on $\mathbb{J}^{2}$ is a diffeomorphism $\psi: \mathbb{J}^{2} \rightarrow \mathbb{J}^{2}$ such that $\psi^{*}\left(\mathcal{J}^{2}\right)=\mathcal{J}^{2}$, and one studies PDEs geometrically by examining the properties of $F^{-1}(0)$ that are invariant under contact transformations. 
By a theorem of Bäcklund [25, Theorem 4.32], every contact transformation on $\mathbb{J}^{2}$ is the prolongation of a contact transformation on $\mathbb{J}^{1}$. Therefore, for any contact transformation $\psi$, there exist functions $A_{i j}, B_{i j}, C_{i j}, D_{i j}$, $a_{i}, m_{i}, \hat{a}_{i}, \hat{m}_{i}$ and $c$, (written as four matrices, four column vectors and a scalar) such that

$$
\psi^{*}\left(\begin{array}{l}
d \xi \\
d z \\
d p
\end{array}\right)=\left(\begin{array}{ccc}
B & \hat{m} & C \\
m^{t} & c & a^{t} \\
A & \hat{a} & D
\end{array}\right)\left(\begin{array}{l}
d \xi \\
d z \\
d p
\end{array}\right)
$$

The assumption that $\psi$ is a contact transformation implies that the symplectic form $\sigma=d p_{1} \wedge d \xi^{1}+d p_{2} \wedge d \xi^{2}+d p_{3} \wedge d \xi^{3}$ is preserved up to scale, so $\psi^{*}(\sigma)=\lambda \sigma, \lambda \neq 0$. One can compute $\tilde{U}_{i j}=\psi^{*}\left(U_{i j}\right)$ by noting that $\psi^{*}\left(d p_{i}-U_{i j} d \xi^{j}\right) \equiv 0$ modulo $\mathcal{J}^{2}$, so in matrix notation

$$
\begin{aligned}
\psi^{*}(d p)-\tilde{U} \psi^{*}(d \xi) & =(A d \xi+\hat{a} d z+D d p)-\tilde{U}(B d \xi+\hat{m} d z+C d p) \\
& \equiv\left(A+\hat{a} p^{t}+D U\right) d \xi-\tilde{U}\left(B+\hat{m} p^{t}+C U\right) d \xi
\end{aligned}
$$

In particular, under the non-degeneracy assumption $\psi^{*}\left(d \xi^{1} \wedge d \xi^{2} \wedge d \xi^{3}\right) \neq$ 0 , this yields

$$
\tilde{U}=\left(A+\hat{a} p^{t}+D U\right)\left(B+\hat{m} p^{t}+C U\right)^{-1}
$$

For this article, consider only PDEs on $u: \mathbb{R}^{3} \rightarrow \mathbb{R}$ of the form

$$
F\left(u_{11}, u_{12}, u_{13}, u_{22}, u_{23}, u_{33}\right)=0
$$

Instead of considering the orbit of this PDE under all contact transformations, consider only those contact transformations that preserve the class of Hessian-only equations, those of the form in Equation (1.7). That is, consider only those contact transformations $\psi$ such that $\psi^{*}\left(d U_{i j}\right) \equiv 0$ modulo $\left\{d U_{k l}\right\}$. Then it must be that $\hat{a}=\hat{m}=0$ in Equation (1.4). Thus, the contact transformations that preserve the Hessian-only form of Equation (1.7) are elements of the conformal symplectic group,

$$
\begin{aligned}
& C S p(3)=\left\{g \in \mathrm{GL}(6, \mathbb{R}): \sigma(g v, g w)=\lambda \sigma(v, w) \forall v, w\left(\text { any } \lambda \in \mathbb{R}^{*}\right)\right\} \\
& =\left\{g=\left(\begin{array}{ll}
B & C \\
A & D
\end{array}\right): 0=A^{t} B-B^{t} A=D^{t} C-C^{t} D,\right. \\
& \left.\lambda I_{3}=D^{t} B-C^{t} A\right\} .
\end{aligned}
$$


In the most general case, the completed domain of all Hessian-only $F$ 's is the Lagrangian Grassmannian,

$$
\Lambda=\mathbb{J}^{2} / \mathbb{J}^{1}=\left\{U \in \operatorname{Gr}_{3}\left(\mathbb{R}^{6}\right):\left.\sigma\right|_{U}=0\right\}
$$

with local coordinates given, for example, by its non- $\xi$-vertical open subset

$$
\Lambda^{o}=\left\{U \in \Lambda:\left.d \xi^{1} \wedge d \xi^{2} \wedge d \xi^{3}\right|_{U} \neq 0\right\}
$$

The conformal symplectic group forms a bundle, $\Pi: C S p(3) \rightarrow \Lambda$. In fact, when considering local transformations of PDEs, only the transformations in $C S p(3)^{o}=\Pi^{-1}\left(\Lambda^{o}\right)$ are permissible, as the condition $d \xi^{1} \wedge d \xi^{2} \wedge$ $d \xi^{3} \neq 0$ must be preserved. As above, one could eliminate the conformal factor and consider only $\operatorname{Sp}(3)^{\circ}$ actions by projectivizing, but there is little utility in doing so.

To be explicit regarding the bundle, suppose $U \in \Lambda^{\circ}$. The open manifold $\Lambda^{o}$ has coordinates given by the components of symmetric matrices, $U_{i j}=$ $U_{j i}$, and $d p_{i} \equiv U_{i j} d \xi^{j}$ modulo $\mathcal{J}^{2}$. Then

$$
\psi^{*}\left(\begin{array}{l}
d \xi \\
d p
\end{array}\right)=\left(\begin{array}{ll}
B & C \\
A & D
\end{array}\right)\left(\begin{array}{l}
d \xi \\
d p
\end{array}\right) .
$$

The assumption that $d \xi$ and $\psi^{*}(d \xi)$ both have maximum rank when pulled back to $N$ implies that $(B+C U)$ is non-singular. Moreover, Equation (1.11) shows that $C S p(3)^{\circ}$ acts on $\Lambda^{o}$ by

$$
g: U \mapsto g(U)=(A+D U)(B+C U)^{-1} .
$$

The fiber subgroup over $U \in \Lambda^{\circ}$ is the stabilizer of $U$, so

$$
\Pi^{-1}(U)=\left\{g: U=g(U)=(A+D U)(B+C U)^{-1}\right\} \cong\{g: A=0\} .
$$

Because $I_{6}$ is in the fiber over $0 \in \Lambda^{o}$, the projection $\Pi: C S p(3)^{o} \rightarrow \Lambda^{o}$ can be computed from Equation (1.12) as

$$
\Pi: g \mapsto g(0)=A B^{-1} .
$$

The most important fact about Equation (1.7) is that $M=F^{-1}(0)$ admits a natural GL(2)-structure wherever the PDE is hyperbolic. Recall that a second-order PDE is called hyperbolic if its leading symbol is a nondegenerate matrix with split signature, and this property is invariant under contact transformations. A related notion of hyperbolicity for hyperplanes 
sitting in projective space is needed to describe the induced GL(2)-structure. The complex Veronese variety is the 2-dimensional projective variety

$$
\left\{\left[Z_{1} Z_{1}: Z_{1} Z_{2}: Z_{1} Z_{3}: Z_{2} Z_{3}: Z_{3} Z_{3}\right], Z \in \mathbb{C P}^{2}\right\} \subset \mathbb{C P}^{5}
$$

The intersection of a generic hyperplane with the Veronese variety is a 1-dimensional rational normal curve, and this curve is uniquely given by such an intersection. The real case needed for the present PDE theory requires a bit more detail to describe accurately. Consider $\mathbb{R P}^{5}$ with coordinates $\left[W_{1}: \cdots: W_{6}\right], \mathbb{R P}^{3}$ with coordinates $\left[Z_{1}: Z_{2}: Z_{3}\right]$ and the Veronese variety defined over $\mathbb{R}$ as above. A generic hyperplane in $\mathbb{R P}^{5}$ is defined uniquely (up to scale and sign) by a single equation

$$
a_{11} W_{1}+a_{12} W_{2}+a_{13} W_{3}+a_{22} W_{4}+a_{23} W_{5}+a_{33} W_{6}=0
$$

The intersection of this hyperplane with the Veronese variety yields an equation on $\mathbb{R}^{3}$ :

$$
a_{11}\left(Z_{1}\right)^{2}+a_{12} Z_{1} Z_{2}+a_{13} Z_{1} Z_{3}+a_{22}\left(Z_{2}\right)^{2}+a_{23} Z_{2} Z_{3}+a_{33}\left(Z_{3}\right)^{2}=0
$$

Depending on the (real) coefficients $a_{i j}$, Equation (1.17) may or may not have real solutions $Z$. If Equation (1.17) has real solutions, then the deprojectivized solution in $\mathbb{R}^{3}$ is a real quadric surface. This quadric may or may not be degenerate. The existence of real non-degenerate solutions is an open condition on the hyperplane in the topology of $\operatorname{Gr}_{5}\left(\mathbb{R}^{6}\right)$. If this condition is satisfied, the hyperplane defined by $\left\{a_{i j}\right\}$ is called hyperbolic. The precise algebraic condition for hyperbolicity is that the real symmetric matrix $\left(a_{i j}\right)$ is non-singular and has split signature [36]. Using the identifications $\mathbb{R}^{6}=\operatorname{Gr}_{5}\left(\mathbb{R}^{6}\right)=\operatorname{Sym}^{2}\left(\mathbb{R}^{3}\right)$, the cone over the Veronese variety (Veronese cone) is identified with the space of symmetric matrices $\left(a_{i j}\right)$ such that $\operatorname{rank}\left(a_{i j}\right) \leq 1$. A hyperbolic hyperplane in $\mathbb{R}^{6}$ intersects the Veronese cone in a rational normal cone, and every rational normal cone in $\mathbb{R}^{6}$ can be written (uniquely, up to scale) this way [20].

Lemma 1.2. Let $F: \Lambda^{o} \rightarrow \mathbb{R}$ be a smooth function, and suppose $U \in \Lambda^{o}$ such that $F(U)=0, d F_{U} \neq 0$, and $\operatorname{ker}\left(d F_{U}\right)$ is hyperbolic as a hyperplane in $\mathbf{T}_{U} \Lambda^{o}=\operatorname{Sym}^{2}\left(\mathbb{R}^{3}\right)$. Then there is an open 5-dimensional submanifold $M \subset$ $\Lambda^{\circ}$ defined by $\left.F\right|_{M}=0$ in a neighborhood of $U$, and $M$ admits a distribution C of rational normal cones. That is, $M$ admits a GL(2)-structure. 
Proof. Aside from the condition on hyperbolicity of the tangent space, the lemma is simply a statement of the implicit function theorem. Here is an explanation of hyperbolicity and its relation to GL(2)-structures.

Fix a coframe $s$ on $\Lambda^{o}$ such that $s_{U}: \mathbf{T}_{U} \Lambda^{o} \rightarrow \operatorname{Sym}^{2}\left(\mathbb{R}^{n}\right)$. (An obvious choice is $s=d U$, using the symmetric matrices as coordinates for $\Lambda^{o}$.) So, there is a distribution of 3 -dimensional Veronese cones defined by $\left\{P \in \mathbf{T} \Lambda^{o}\right.$ : rank $s(P) \leq 1\}$. This distribution of cones is $C S p(3)$-invariant, for in the first part of $[9$, Theorem XX], Cartan proves that the above bundle $C S p(3)$ is exactly the frame bundle over $\Lambda$ whose action on $\mathbf{T}_{U} \Lambda \simeq \operatorname{Sym}^{2}\left(\mathbb{R}^{n}\right)$ is to act irreducibly on the coefficients of the equation of a cone, for example Equation (1.17).

In the context of the lemma, Equation (1.16) describes the intersection of $\operatorname{ker}\left(d F_{U}\right)$ with the Veronese cone, so the symmetric matrix $\left(a_{i j}\right)$ is, up to scale, the leading symbol of $F$ at $U$. Therefore, if $U$ is a regular point for the regular value 0 of $F: \Lambda^{o} \rightarrow \mathbb{R}$ and if the PDE $F=0$ is hyperbolic at $U$, then $F^{-1}(0)$ admits a field of rational normal cones near $U$ given by these intersections.

The fact that every hyperbolic PDE of the form in Equation (1.7) admits a GL(2)-structure would only be an algebraic curiosity, except that the integrability of the PDE is intimately related to the 2,3-integrability of the GL(2)-structure.

Theorem 1.1 [14, Theorem 3]. Fix a hyperbolic PDE of the form in Equation (1.7) and its corresponding $\mathrm{GL}(2)$-structure $\pi: \mathcal{B} \rightarrow M$. Then $\mathcal{B}$ is 2-integrable. Moreover, the PDE is integrable via 3-parameter hydrodynamic reductions if and only if $\mathcal{B}$ is also 3-integrable.

Theorem 1.1 as presented in [14] apparently requires that, for each 3-secant $N^{3} \subset M^{5}$, the characteristic net defined by the intersection $\mathbf{T} N \cap \mathbf{C}$ is a coordinate net. According to Corollary 4.1, this requirement is redundant.

As discussed in [14], for PDEs in three independent variables, the existence of 3-parameter hydrodynamic reductions parametrized by three functions of one variable implies the existence of $k$-parameter hydrodynamic reductions parametrized by $k$ functions of one variable for all $k \geq 3$.

Corollary 1.1. $F\left(u_{i j}\right)=0$ is integrable via $k$-parameter hydrodynamic reductions for all $k \geq 2$ if and only if the induced GL(2)-structure over $F^{-1}(0)$ is 2,3-integrable. 
Definition 1.3 (Hessian hydrodynamic PDEs). A PDE on $u: \mathbb{R}^{3} \rightarrow \mathbb{R}$ is Hessian hydrodynamic if and only if it is hyperbolic, is of the form $F\left(u_{11}, \ldots, u_{33}\right)=0$, and is integrable by means of 3 -parameter hydrodynamic reductions.

The remainder of this article is a study of Hessian hydrodynamic PDEs via their associated 2,3-integrable GL(2)-structures.

\section{A preferred connection}

In this section, Cartan's method of equivalence is applied to GL(2)structures. Cartan's method of equivalence is a standard tool in the field of exterior differential systems; it is an algorithm for finding all of the differential invariants of a geometric structure, and the first step is to fix a preferred connection among all the possible pseudo-connections of a geometric structure $[4,19,22]$. (The distinction between a connection and a pseudoconnection is simply whether it was obtained via such an algorithm.) The result of the algorithm is a preferred global coframe for the GL(2)-structure that splits into the semi-basic "tautological" form, $\omega$, and the vertical $\mathfrak{g l}(2)$ valued "connection" form, $\theta$.

For a $\mathrm{GL}(2)$-structure $\pi: \mathcal{B} \rightarrow M$, let $\omega$ denote the tautological 1-form defined by $\omega_{b}=b \circ \pi_{*}: \mathbf{T}_{b} \mathcal{B} \rightarrow \mathcal{V}_{4}$. As always, $\omega$ is a globally defined 1 -form on $\mathcal{B}$ with linearly independent components, and it is semi-basic, meaning $\left.\omega\right|_{\text {ker } \pi_{*}}=0$. A pseudo-connection on $\mathcal{B}$ is a 1 -form $\theta$ taking values in the nontrivial representation of $\mathfrak{g l}(2)$ in $\mathfrak{g l}\left(\mathcal{V}_{4}\right)$ such that $d \omega=-\theta \wedge \omega+T(\omega \wedge \omega)$ for some torsion $T(b): \mathcal{V}_{4} \wedge \mathcal{V}_{4} \rightarrow \mathcal{V}_{4}$.

The goal is to fix a particular $\theta$ that minimizes the torsion $T$. To carry out this process, one needs to understand the $\mathrm{SL}(2)$ representation theory on $\mathcal{V}_{n}$. The decomposition of the tensor product $\mathcal{V}_{m} \otimes \mathcal{V}_{n}$ into irreducible components is

$$
\mathcal{V}_{m} \otimes \mathcal{V}_{n}=\mathcal{V}_{|m-n|} \oplus \mathcal{V}_{|m-n|+2} \oplus \cdots \oplus \mathcal{V}_{m+n-2} \oplus \mathcal{V}_{m+n}
$$

The projections onto the various components are given by the ClebschGordan $[5,21]$ pairings $\langle\cdot, \cdot\rangle_{p}: \mathcal{V}_{m} \otimes \mathcal{V}_{n} \rightarrow \mathcal{V}_{m+n-2 p}$, which are provided by the formula

$$
\langle u, v\rangle_{p}=\frac{1}{p !} \sum_{k=0}^{p}(-1)^{k}\left(\begin{array}{l}
p \\
k
\end{array}\right) \frac{\partial^{p} u}{\partial x^{p-k} \partial y^{k}} \cdot \frac{\partial^{p} v}{\partial x^{k} \partial y^{p-k}}
$$


This pairing has some important properties. Notice that $\langle u, v\rangle_{p}=$ $(-1)^{p}\langle v, u\rangle_{p}$ and that the pairing is non-trivial for $0 \leq p \leq \min \{m, n\}$. Hence, the tensor decomposition can be further refined in terms of the symmetric and alternating tensors:

$$
\begin{aligned}
& \mathcal{V}_{n} \circ \mathcal{V}_{n}=\mathcal{V}_{2 n} \oplus \mathcal{V}_{2 n-4} \oplus \cdots \oplus \mathcal{V}_{0 \text { or } 2} \\
& \mathcal{V}_{n} \wedge \mathcal{V}_{n}=\mathcal{V}_{2 n-2} \oplus \mathcal{V}_{2 n-6} \oplus \cdots \oplus \mathcal{V}_{2 \text { or } 0}
\end{aligned}
$$

Notice too that $\langle\cdot, \cdot\rangle_{n}: \mathcal{V}_{n} \otimes \mathcal{V}_{n} \rightarrow \mathcal{V}_{0}=\mathbb{R}$ is a non-degenerate symmetric or skew bilinear form. Hence, for fixed $u \in \mathcal{V}_{n}$ the map $\langle u, \cdot\rangle_{n}: \mathcal{V}_{n} \rightarrow \mathcal{V}_{0}=\mathbb{R}^{1}$ provides a natural identification, $\mathcal{V}_{n}=\mathcal{V}_{n}^{*}$, and one need never distinguish between dual spaces when considering representations.

For any derivation over $\mathbb{R}[x, y]$, a Leibniz rule over the pairing holds. Because $\mathrm{SL}(2)$ is infinitesimally generated by $X, Y$ and $H$, this means that the pairings are $\mathrm{SL}(2)$-equivariant. That is, $\alpha\left(\langle u, v\rangle_{p}\right)=\langle\alpha(u), v\rangle_{p}+$ $\langle u, \alpha(v)\rangle_{p}$ for any $\alpha \in \mathfrak{s l}(2)$, which implies that $a \cdot\langle u, v\rangle_{p}=\langle a \cdot u, a \cdot v\rangle_{p}$ for any $a \in \mathrm{SL}(2)$. The pairing is not $\mathrm{GL}(2)$-equivariant, but the scaling action is easily computed easily where required. The geometric objects encountered here are projectively defined, so this variance in scaling is of little concern.

Most importantly, the pairing can be generalized to binary-polynomialvalued differential forms over a manifold. If $u \in \Gamma\left(\wedge^{r} \mathbf{T}^{*} \mathcal{B} \otimes \mathcal{V}_{m}\right)$ and $v \in$ $\Gamma\left(\wedge^{s} \mathbf{T}^{*} \mathcal{B} \otimes \mathcal{V}_{n}\right)$, then extend the definition by using the wedge product:

$$
\langle u, v\rangle_{p}=\frac{1}{p !} \sum_{k=0}^{p}(-1)^{k}\left(\begin{array}{l}
p \\
k
\end{array}\right) \frac{\partial^{p} u}{\partial x^{p-k} \partial y^{k}} \wedge \frac{\partial^{p} v}{\partial x^{k} \partial y^{p-k}}
$$

In this generalization, the symmetry of the pairing is further altered by the degree of the forms: $\langle u, v\rangle_{p}=(-1)^{r s+p}\langle v, u\rangle_{p}$. If $\lambda$ is an $\mathbb{R}$-valued 1 -form acting by the scaling action $\lambda I_{m}$ on $\mathcal{V}_{m}$, then $\lambda \wedge u$ may be written as the trivial pairing $\langle\lambda, u\rangle_{0}=(-1)^{r}\langle u, \lambda\rangle_{0} \in \Gamma\left(\wedge^{r+1} \mathbf{T}^{*} \mathcal{B} \otimes \mathcal{V}_{m}\right)$.

As $\mathrm{SL}(2)$ representations, $\mathfrak{g l}(2)=\mathfrak{s l}(2) \oplus \mathbb{R}=\mathcal{V}_{2} \oplus \mathcal{V}_{0}$. Thus, a $\mathfrak{g l}(2)$ valued pseudo-connection $\theta$ decomposes as $(\varphi, \lambda)$ with $\varphi \in \Gamma\left(\mathbf{T}^{*} \mathcal{B} \otimes \mathcal{V}_{2}\right)$ and $\lambda \in \Gamma\left(\mathbf{T}^{*} \mathcal{B} \otimes \mathcal{V}_{0}\right)$. The torsion of a generic $\theta$ is

$$
\begin{aligned}
T: \mathcal{B} \rightarrow \mathcal{V}_{4} \otimes\left(\mathcal{V}_{4}^{*} \wedge \mathcal{V}_{4}^{*}\right) & =\mathcal{V}_{4} \otimes\left(\mathcal{V}_{2} \oplus \mathcal{V}_{6}\right) \\
& =\left(\mathcal{V}_{2} \oplus \mathcal{V}_{4} \oplus \mathcal{V}_{6}\right) \oplus\left(\mathcal{V}_{2} \oplus \mathcal{V}_{4} \oplus \mathcal{V}_{6} \oplus \mathcal{V}_{8} \oplus \mathcal{V}_{10}\right)
\end{aligned}
$$

SO

$$
T=\left(T_{2}^{2}+T_{4}^{2}+T_{6}^{2}\right)+\left(T_{2}^{6}+T_{4}^{6}+T_{6}^{6}+T_{8}^{6}+T_{10}^{6}\right)
$$


In this notation, each $T_{n}^{r}$ is a distinct irreducible component of $T$. The lower index $n$ indicates the weight of the representation in which $T_{n}^{r}$ takes values, and the upper index $r$ indicates the summand from which it was obtained.

Thus, Cartan's first structure equation for a GL(2)-structure may be written in either vector form or polynomial form:

$$
\begin{aligned}
d \omega= & -\theta \wedge \omega+T(\omega \wedge \omega) \\
= & -\langle\varphi, \omega\rangle_{1}-\langle\lambda, \omega\rangle_{0}+\left\langle T_{2}^{2},\langle\omega, \omega\rangle_{3}\right\rangle_{0}+\left\langle T_{4}^{2},\langle\omega, \omega\rangle_{3}\right\rangle_{1}+\left\langle T_{6}^{2},\langle\omega, \omega\rangle_{3}\right\rangle_{2} \\
& +\left\langle T_{2}^{6},\langle\omega, \omega\rangle_{1}\right\rangle_{2}+\left\langle T_{4}^{6},\langle\omega, \omega\rangle_{1}\right\rangle_{3}+\left\langle T_{6}^{6},\langle\omega, \omega\rangle_{1}\right\rangle_{4} \\
& +\left\langle T_{8}^{6},\langle\omega, \omega\rangle_{1}\right\rangle_{5}+\left\langle T_{10}^{6},\langle\omega, \omega\rangle_{1}\right\rangle_{6} .
\end{aligned}
$$

Explicitly, the connection term is

$$
\theta \wedge \omega=\left(\begin{array}{ccccc}
-8 \varphi_{0}+\lambda & 8 \varphi_{-2} & 0 & 0 & 0 \\
-2 \varphi_{2} & -4 \varphi_{0}+\lambda & 6 \varphi_{-2} & 0 & 0 \\
0 & -4 \varphi_{2} & \lambda & 4 \varphi_{-2} & 0 \\
0 & 0 & -6 \varphi_{2} & 4 \varphi_{0}+\lambda & 2 \varphi_{-2} \\
0 & 0 & 0 & -8 \varphi_{2} & 8 \varphi_{0}+\lambda
\end{array}\right) \wedge\left(\begin{array}{c}
\omega^{-4} \\
\omega^{-2} \\
\omega^{0} \\
\omega^{2} \\
\omega^{4}
\end{array}\right)
$$

so the matrix representation of $\theta$ is $\left(2 \varphi_{-2} X-2 \varphi_{0} H+2 \varphi_{2} Y+\lambda I_{5}\right)$, which takes values in $\mathfrak{g l}(2) \subset \mathfrak{g l}\left(\mathcal{V}_{4}\right)$.

Theorem 2.1. A generic GL(2)-structure $\pi: \mathcal{B} \rightarrow M$ admits a twodimensional family of connections such that the essential torsion $T$ decomposes irreducibly as

$$
T=T_{2}+T_{6}+T_{8}+T_{10} \in \mathcal{V}_{2} \oplus \mathcal{V}_{6} \oplus \mathcal{V}_{8} \oplus \mathcal{V}_{10} \subset \mathcal{V}_{4} \otimes\left(\mathcal{V}_{4}^{*} \wedge \mathcal{V}_{4}^{*}\right)
$$

The two-dimensional family is parametrized by the possible equivariant inclusions of $\mathcal{V}_{2} \oplus \mathcal{V}_{6} \oplus \mathcal{V}_{8} \oplus \mathcal{V}_{10}$ into $\mathcal{V}_{4} \otimes\left(\mathcal{V}_{4}^{*} \wedge \mathcal{V}_{4}^{*}\right)$, and once such an inclusion is chosen, the connection is unique.

Proof. Changes of pseudo-connection are of the form $\hat{\varphi}=\varphi+P(\omega)$ and $\hat{\lambda}=$ $\lambda+Q(\omega)$ where $P \in \mathcal{V}_{2} \otimes \mathcal{V}_{4}^{*}=\mathcal{V}_{2} \oplus \mathcal{V}_{4} \oplus \mathcal{V}_{6}$ and $Q \in \mathcal{V}_{0} \otimes \mathcal{V}_{4}^{*}=\mathcal{V}_{4}$. Preferred connections are obtained by analyzing the skewing map $\delta$ that 
describes how changes of pseudo-connection (equivalently, changes of horizontal section of the frame bundle) affect the torsion [4]:

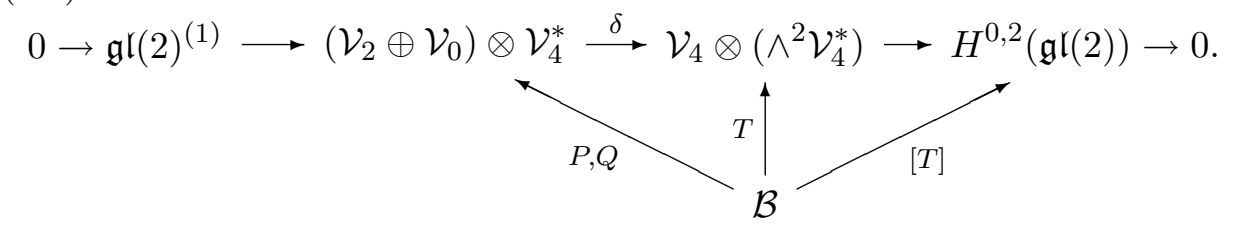

For current purposes, $\mathfrak{g l}(2)^{(1)}$ and $H^{0,2}(\mathfrak{g l}(2))$ are defined by the exactness of the sequence [19]. They depend on the representation of the group GL(2), in this case the irreducible representation $\mathcal{V}_{4}$. To find the space of essential torsion, $H^{0,2}(\mathfrak{g l}(2))$, one must compute $\delta P$ and $\delta Q$.

Fix $P \in \mathcal{V}_{2} \oplus \mathcal{V}_{4} \oplus \mathcal{V}_{6}$, where $\mathcal{V}_{2} \ni P(\omega)=\left\langle P_{2}, \omega\right\rangle_{2}+\left\langle P_{4}, \omega\right\rangle_{3}+\left\langle P_{6}, \omega\right\rangle_{4}$. Let $\delta P \in \mathcal{V}_{4} \otimes \wedge^{2}\left(\mathcal{V}_{4}\right)$ have components $\delta P=\delta P_{2}^{2}+\delta P_{4}^{2}+\delta P_{6}^{2}+\delta P_{2}^{6}+$ $\delta P_{4}^{6}+\delta P_{6}^{6}+\delta P_{8}^{6}+\delta P_{10}^{6}$, similar to the decomposition of $T$. Since these are irreducible components and the action of $\delta$ must be $\mathrm{SL}(2)$-equivariant, Schur's lemma implies $\delta$ must preserve the weights of the representations. In particular there must exist constants $a_{2}, a_{4}, a_{6}, b_{2}, b_{4}$ and $b_{6}$ such that $\delta P_{2}^{2}=a_{2} P_{2}, \delta P_{2}^{6}=b_{2} P_{2}$ and so on. Thus,

$$
\begin{aligned}
0 & =\langle P(\omega), \omega\rangle_{1}-\delta P(\omega, \omega) \\
& =\left\langle\left\langle P_{2}, \omega\right\rangle_{2}, \omega\right\rangle_{1}+\left\langle\left\langle P_{4}, \omega\right\rangle_{3}, \omega\right\rangle_{1}+\left\langle\left\langle P_{6}, \omega\right\rangle_{4}, \omega\right\rangle_{1} \\
& -\left\langle a_{2} P_{2},\langle\omega, \omega\rangle_{3}\right\rangle_{0}-\left\langle a_{4} P_{4},\langle\omega, \omega\rangle_{3}\right\rangle_{1}-\left\langle a_{6} P_{6},\langle\omega, \omega\rangle_{3}\right\rangle_{2} \\
& -\left\langle b_{2} P_{2},\langle\omega, \omega\rangle_{1}\right\rangle_{2}-\left\langle b_{4} P_{4},\langle\omega, \omega\rangle_{1}\right\rangle_{3}-\left\langle b_{6} P_{6},\langle\omega, \omega\rangle_{1}\right\rangle_{4}
\end{aligned}
$$

Carrying out this computation shows that

$$
a_{2}=\frac{3}{10}, \quad b_{2}=\frac{1}{5}, \quad a_{4}=\frac{1}{2}, \quad b_{4}=0, \quad a_{6}=-\frac{1}{5}, \quad b_{6}=-\frac{1}{20} .
$$

Similarly, fix $Q \in \mathcal{V}_{4}$, where $\mathcal{V}_{0} \ni Q(\omega)=\left\langle Q_{4}, \omega\right\rangle_{4}$. Let $\delta Q \in \mathcal{V}_{4} \otimes \wedge^{2}\left(\mathcal{V}_{4}\right)$ have components $\delta Q=\delta Q_{2}^{2}+\delta Q_{4}^{2}+\delta Q_{6}^{2}+\delta Q_{2}^{6}+\delta Q_{4}^{6}+\delta Q_{6}^{6}+\delta Q_{8}^{6}+$ $\delta Q_{10}^{6}$, but again the image must have the same weight as the domain. In particular, there must exist constants $c_{4}$ and $d_{4}$ such that

$$
\begin{aligned}
0 & =\langle Q(\omega), \omega\rangle_{0}-\delta Q(\omega, \omega) \\
& =\left\langle\left\langle Q_{4}, \omega\right\rangle_{4}, \omega\right\rangle_{0}-\left\langle c_{4} Q_{4},\langle\omega, \omega\rangle_{3}\right\rangle_{1}-\left\langle d_{4} Q_{4},\langle\omega, \omega\rangle_{1}\right\rangle_{3}
\end{aligned}
$$


Carrying out this computation shows that

$$
c_{4}=-\frac{1}{40}, \quad d_{4}=-\frac{1}{160} .
$$

Fix a generic pseudo-connection $(\varphi, \lambda)$ with torsion $T$. Consider another pseudo-connection $\hat{\varphi}=\varphi+P(\omega), \hat{\lambda}=\lambda+Q(\lambda)$ with torsion $\hat{T}$. Then

$$
\begin{aligned}
\hat{T}(\omega, \omega) & =d \omega+\langle\hat{\varphi}, \omega\rangle_{1}+\langle\hat{\lambda}, \omega\rangle_{0} \\
& =d \omega+\langle\varphi, \omega\rangle_{1}+\langle P(\omega), \omega\rangle_{1}+\langle Q(\omega), \omega\rangle_{0} \\
& =(T+\delta P+\delta Q)(\omega, \omega) .
\end{aligned}
$$

Using Equation (2.11) and Equation (2.13), the absorption of torsion is dictated by the solvability of the following equations in terms of $P$ and $Q$ for fixed $T$ and $\hat{T}$ :

$$
\begin{aligned}
\hat{T}_{2}^{2} & =T_{2}^{2}+\frac{3}{10} P_{2} \\
\hat{T}_{4}^{2} & =T_{4}^{2}+\frac{1}{2} P_{4}-\frac{1}{40} Q_{4} \\
\hat{T}_{6}^{2} & =T_{6}^{2}-\frac{1}{5} P_{6} \\
\hat{T}_{2}^{6} & =T_{2}^{6}+\frac{1}{5} P_{2} \\
\hat{T}_{4}^{6} & =T_{4}^{6}-\frac{1}{160} Q_{4} \\
\hat{T}_{6}^{6} & =T_{6}^{6}-\frac{1}{20} P_{6} \\
\hat{T}_{8}^{6} & =T_{8}^{6} \\
\hat{T}_{10}^{6} & =T_{10}^{6} .
\end{aligned}
$$

Generally, one may choose $P_{2}$ to force exactly one linear combination of $\hat{T}_{2}^{6}$ and $\hat{T}_{2}^{2}$ to vanish. Similarly, one may choose $P_{6}$ to force exactly one linear combination of $\hat{T}_{6}^{6}$ and $\hat{T}_{6}^{2}$ to vanish. Unique $Q_{4}$ and $P_{4}$ eliminate $\hat{T}_{4}^{6}$ and $\hat{T}_{4}^{2}$. All other components of $\hat{T}$ are fixed. Thus, $\mathfrak{g l}(2)^{(1)}=\mathfrak{s l}(2)^{(1)}=$ 0 and $H^{0,2}(\mathfrak{s l}(2))=\mathcal{V}_{2} \oplus \mathcal{V}_{4} \oplus \mathcal{V}_{6} \oplus \mathcal{V}_{8} \oplus \mathcal{V}_{10}$, while $H^{0,2}(\mathfrak{g l}(2))=\mathcal{V}_{2} \oplus \mathcal{V}_{6} \oplus$ $\mathcal{V}_{8} \oplus \mathcal{V}_{10}$ 
Corollary 2.1 (Preferred GL(2) connection). A GL(2)-structure $\mathcal{B} \rightarrow M$ admits a unique connection $\varphi, \lambda$ such that $\mathcal{B}$ has first structure equation

$$
\begin{aligned}
d \omega= & -\langle\varphi, \omega\rangle_{1}-\langle\lambda, \omega\rangle_{0} \\
& +\left\langle T_{2},\langle\omega, \omega\rangle_{1}\right\rangle_{2}+\left\langle T_{6},\langle\omega, \omega\rangle_{1}\right\rangle_{4} \\
& +\left\langle T_{8},\langle\omega, \omega\rangle_{1}\right\rangle_{5}+\left\langle T_{10},\langle\omega, \omega\rangle_{1}\right\rangle_{6} .
\end{aligned}
$$

Proof. Of the possible connections, choose the one that absorbs $T_{2}^{2}$ and $T_{6}^{2}$. The remaining essential torsion is $T=T_{2}^{6}+T_{6}^{6}+T_{8}^{6}+T_{10}^{6}$.

Henceforth, all references to $\theta, \varphi, \lambda$ and $T$ assume the connection in Corollary 2.1. The specification of this connection over the others is arbitrary, but it does not affect any subsequent theorems in this article, since the $\mathcal{V}_{2}$ and $\mathcal{V}_{6}$ components of essential torsion turn out to be unimportant in the study of integrable PDEs.

\section{2-Integrability}

Theorem 3.1. If a $\mathrm{GL}(2)$-structure $\pi: \mathcal{B} \rightarrow M$ is 2-integrable, then $T=$ $T_{8} \quad$ (that is, $\left.T_{2}=T_{6}=T_{10}=0\right)$, and the bi-secant surfaces in $M$ are parametrized by two functions of one variable. Conversely, if $\mathcal{B}$ is a smooth $\mathrm{GL}(2)$-structure with $T=T_{8}$, then $\mathcal{B}$ is 2-integrable.

Proof. To prove the theorem, one must find the conditions on $\mathcal{B}$ that allow an arbitrary bi-secant plane $E \in \mathrm{Gr}_{2}\left(\mathbf{T}_{p} M\right)$ to be extended to a bi-secant surface $N \subset M$.

In a neighborhood $M^{\prime}$ of $p$, Fix $u: M^{\prime} \rightarrow \mathcal{B}\left(M^{\prime}\right)$, a section of $\pi: \mathcal{B} \rightarrow M$; that is, fix $u$, a $\mathrm{GL}(2)$ coframe on $M^{\prime}$. Let $b=u(p)$. Since $E$ is bi-secant, $b(E) \subset \mathcal{V}_{4}$ is spanned by $\left(g_{1} x-h_{1} y\right)^{4}$ and $\left(g_{2} x-h_{2} y\right)^{4}$ with $g_{1} h_{2} \neq g_{2} h_{1}$. Through a GL(2) frame adaptation redefining $u$, one may assume that $b(E)=\operatorname{span}\left\{x^{4}, y^{4}\right\} \subset \mathcal{V}_{4} . \quad$ Let $\quad \tilde{E}=u_{*}(E) \in \operatorname{Gr}_{2}\left(\mathbf{T}_{b} \mathcal{B}\right)$. Then $\omega^{-4} \wedge$ $\left.\omega^{4}\right|_{\tilde{E}} \neq 0$.

Consider the linear Pfaffian exterior differential system $\mathcal{I}$ differentially generated by the 1 -forms $\left\{\omega^{-2}, \omega^{0}, \omega^{2}\right\}$ with the independence condition $\omega^{-4} \wedge \omega^{4} \neq 0$. It suffices to prove the existence of a surface $\tilde{N} \subset \mathcal{B}$ that is integral to $\mathcal{I}$, because $u\left(\pi_{*}(\mathbf{T} \tilde{N})\right)=\omega_{u}(\mathbf{T} \tilde{N})=\operatorname{span}\left\{x^{4}, y^{4}\right\}$ implies that the surface $N=\pi(\tilde{N}) \subset M^{\prime}$ is bi-secant. In fact, by adapting the moving coframe $u$ appropriately, every bi-secant surface through $E$ must arise this way. 
The generating 2-forms of $\mathcal{I}$ are

$$
d\left(\begin{array}{c}
\omega^{-2} \\
\omega^{0} \\
\omega^{2}
\end{array}\right) \equiv\left(\begin{array}{cc}
-2 \varphi_{2} & 0 \\
0 & 0 \\
0 & 2 \varphi_{-2}
\end{array}\right) \wedge\left(\begin{array}{c}
\omega^{-4} \\
\omega^{4}
\end{array}\right)+\left(\begin{array}{c}
\tau^{-2} \\
\tau^{0} \\
\tau^{2}
\end{array}\right) \omega^{-4} \wedge \omega^{4}
$$

modulo $\omega^{-2}, \omega^{0}, \omega^{2}$, where

$$
\begin{aligned}
\tau^{-2} & =48 T_{2,-2}+8640 T_{6,-2}+322,560 T_{8,-2}-4,838,400 T_{10,-2}, \\
\tau^{0} & =-96 T_{2,0}+23,040 T_{6,0}-4,838,400 T_{10,0}, \\
\tau^{2} & =48 T_{2,2}+8640 T_{6,2}-322,560 T_{8,2}-4,838,400 T_{10,2}
\end{aligned}
$$

Because of the independence condition, integral elements exist only when the torsion can be absorbed. The torsion component $\tau^{0}$ can never be absorbed, so integral manifolds exist only when $\tau^{0}=0$. The condition of 2 -integrability means that every 2 -secant plane is tangent to a 2 -secant surface, but the $\mathrm{GL}(2)$ action is transitive on 2 -secant planes in $\mathbf{T}_{p} M$; therefore, 2-integrability implies that $\tau^{0}=0$ for every element in the GL(2) orbit of $T$. Under the GL(2) action, the coordinates of the irreducible representations of $T$ will change, so each irreducible representation that appears in $\tau^{0}$ must vanish identically. Hence, 2 -integrability of $M$ by integral manifolds implies

$$
T_{10}=T_{6}=T_{2}=0
$$

The remaining torsion components, $\tau^{-2}$ and $\tau^{2}$, are absorbed by setting $\pi_{1}=2 \varphi_{2}-322,560 T_{8,-2} \omega^{4}$ and $\pi_{2}=-2 \varphi_{-2}-322,560 T_{8,2} \omega^{-4}$, so

$$
d\left(\begin{array}{c}
\omega^{-2} \\
\omega^{0} \\
\omega^{2}
\end{array}\right) \equiv\left(\begin{array}{cc}
\pi_{1} & 0 \\
0 & 0 \\
0 & \pi_{2}
\end{array}\right) \wedge\left(\begin{array}{c}
\omega^{-4} \\
\omega^{4}
\end{array}\right), \quad \bmod \omega^{-2}, \omega^{0}, \omega^{2}
$$

This proves the torsion condition in the theorem.

Conversely, to establish the existence and parametrization of bi-secant surfaces, one can apply Cartan's test for involutivity to the tableau in Equation (3.4) [3, 22]. For a generic flag of $\mathbf{T}_{p} N$ obtained from generic linear combinations of $\omega^{-4}$ and $\omega^{4}$, the tableau has Cartan characters $s_{1}=2$ and $s_{2}=0$. Let $\tilde{\nu}: \tilde{N} \rightarrow \mathcal{B}$ denote the embedding of the integral surface. The space of integral elements for the $\operatorname{EDS}\left(\mathcal{I}, \omega^{-4} \wedge \omega^{4}\right)$ is 2-dimensional, as parametrized by the coefficients $p_{1,4}$ and $p_{3,-4}$ that appear in the pulledback forms $\tilde{\nu}^{*}\left(\pi_{1}\right)=p_{1,4} \tilde{\nu}^{*}\left(\omega^{4}\right)$ and $\tilde{\nu}^{*}\left(\pi_{2}\right)=p_{3,-4} \tilde{\nu}^{*}\left(\omega^{-4}\right)$. Therefore, if $\pi$ : $\mathcal{B} \rightarrow M$ is 2-integrable, then bi-secant surfaces in $M$ depend on two functions of one variable. With the Cartan characters computed, Cartan's test 
for involutivity applies, so integral surfaces for the linear Pfaffian system exist in the real-analytic category.

More can be said by employing modern theorems regarding hyperbolic exterior differential systems [34]. The characteristic variety of the tableau consists of two real points, and because a generic tableau is involutive with $s_{1}=2$, Yang's generalization of the Cartan-Kähler theorem to smooth hyperbolic systems implies that integral surfaces exist and are parametrized by two functions of one variable in the smooth category [34, Theorem 1.19]. A special case of this observation is revisited in Section 6.1.

By restricting the torsion, Theorem 3.1 provides the necessary and sufficient first-order conditions for 2-integrability. These first-order conditions imply syzygies on the second-order invariants via the Bianchi identity.

Corollary 3.1. Suppose the $\mathrm{GL}(2)$-structure $\pi: \mathcal{B} \rightarrow M$ is 2-integrable with torsion $T$. Let $S=\nabla(T)$ denote the covariant derivative of $T$, and let $Q=T \circ T$ denote the symmetric product of $T$. Then $\mathcal{B}$ has structure equations of the form

$$
\begin{aligned}
d \omega= & -\langle\varphi, \omega\rangle_{1}-\langle\lambda, \omega\rangle_{0}+\left\langle T,\langle\omega, \omega\rangle_{1}\right\rangle_{5} \\
d \varphi= & -\frac{1}{2}\langle\varphi, \varphi\rangle_{1}+\left\langle R_{0}^{2},\langle\omega, \omega\rangle_{3}\right\rangle_{0}+\left\langle 48 Q_{4}+42 S_{4},\langle\omega, \omega\rangle_{3}\right\rangle_{2} \\
& +\left\langle 45 S_{6},\langle\omega, \omega\rangle_{1}\right\rangle_{5}+\left\langle 33 S_{8},\langle\omega, \omega\rangle_{1}\right\rangle_{6}+\left\langle-8 Q_{4}-12 S_{4},\langle\omega, \omega\rangle_{1}\right\rangle_{4} \\
d \lambda= & 960\left\langle S_{6},\langle\omega, \omega\rangle_{1}\right\rangle_{6}
\end{aligned}
$$

for a scalar curvature function $R_{0}^{2}: \mathcal{B} \rightarrow \mathcal{V}_{0}$

Proof. Write $\nabla=d+\theta$ for the covariant derivative on $\mathcal{B}$ defined by the connection 1-form $(\varphi, \lambda)$. Second-order consequences of 2-integrability arise from the Bianchi identity, $\nabla(\theta) \wedge \omega=\nabla(T(\omega \wedge \omega))$. Curvature appears in $\nabla(\theta)$, which splits into $R(\omega \wedge \omega)=d \varphi+\frac{1}{2}\langle\varphi, \varphi\rangle_{1}$ and $r(\omega \wedge \omega)=d \lambda$. The covariant derivative of the torsion two-form, $\nabla(T(\omega \wedge \omega))$, expands as $\nabla(T(\omega \wedge \omega))=\nabla(T)(\omega \wedge \omega)+2 Q(T, T)(\omega \wedge \omega \wedge \omega)$, so the Bianchi identity for a $\mathrm{GL}(2)$-structure is

$$
R(\omega \wedge \omega) \wedge \omega+r(\omega \wedge \omega) \wedge \omega=\nabla(T)(\omega \wedge \omega)+2 Q(T, T)(\omega \wedge \omega \wedge \omega)
$$


Each of $R, r, \nabla(T)$ and $Q$ is a function on $\mathcal{B}$ to the appropriate $\operatorname{SL}(2)$ module:

$$
\begin{aligned}
R & : \mathcal{B} \\
r & : \mathcal{B l}(2) \otimes\left(\mathcal{V}_{4}^{*} \wedge \mathcal{V}_{4}^{*}\right), \\
\nabla(T) & : \mathcal{B} \otimes\left(\mathcal{V}_{4}^{*} \wedge \mathcal{V}_{4}^{*}\right), \\
Q & \left.: \mathcal{B} \rightarrow \operatorname{Sym}^{2}\left(H^{0,2}(\mathfrak{g l}(2)) \otimes \mathcal{V}_{4}^{*}, \text { and }(2)\right)\right) \cap\left(\mathcal{V}_{4} \otimes \wedge^{3} \mathcal{V}_{4}^{*}\right)
\end{aligned}
$$

Since $\mathcal{B}$ is 2-integrable, $T$ takes values only in $\mathcal{V}_{8} \subset H^{0,2}(\mathfrak{g l} l(2))$. Using the Clebsch-Gordan decomposition, the irreducible components of these functions are (omitting the domain $\mathcal{B}$ for brevity)

$$
\begin{aligned}
R & =\left(R_{0}^{2}+R_{2}^{2}+R_{4}^{2}\right)+\left(R_{4}^{6}+R_{6}^{6}+R_{8}^{6}\right) \in \mathcal{V}_{2} \otimes\left(\mathcal{V}_{2} \oplus \mathcal{V}_{6}\right), \\
r & =r_{2}+r_{6} \in \mathcal{V}_{0} \otimes\left(\mathcal{V}_{2} \oplus \mathcal{V}_{6}\right) \\
\nabla T & =S_{4}+S_{6}+S_{8}+S_{10}+S_{12} \in \mathcal{V}_{8} \otimes \mathcal{V}_{4}, \text { and } \\
Q & =Q_{4}+Q_{8} \in \operatorname{Sym}^{2}\left(\mathcal{V}_{8}\right) \cap\left(\mathcal{V}_{4} \otimes\left(\mathcal{V}_{2} \oplus \mathcal{V}_{6}\right)\right)
\end{aligned}
$$

Thus, Equation (3.6) and Schur's lemma together imply linear relations among the irreducible components listed in Equation (3.8). To find these relations, one can expand Equation (3.6) using the Clebsch-Gordan pairing; for example, one of the terms is

$$
\begin{aligned}
\left\langle\nabla T,\langle\omega, \omega\rangle_{1}\right\rangle_{5}= & \left\langle\left\langle S_{4}, \omega\right\rangle_{0}+\left\langle S_{6}, \omega\right\rangle_{1}+\left\langle S_{8}, \omega\right\rangle_{2}+\left\langle S_{10}, \omega\right\rangle_{3}\right. \\
& \left.+\left\langle S_{12}, \omega\right\rangle_{4},\langle\omega, \omega\rangle_{1}\right\rangle_{5}
\end{aligned}
$$

The result is $S_{10}=0, R_{8}^{6}=33 S_{8}, R_{6}^{6}=45 S_{6}, r_{6}=960 S_{6}, R_{4}^{6}=-12 S_{4}-$ $8 Q_{4}, R_{4}^{2}=42 S_{4}+48 Q_{4}, r_{2}=0$ and $R_{2}^{2}=0$. In particular, $R_{0}^{2}$ is the only irreducible component of $r$ and $R$ that is not an algebraic function of $T$ and $\nabla(T)$.

\section{3-Integrability}

Theorem 4.1. If an analytic $\mathrm{GL}(2)$-structure $\pi: \mathcal{B} \rightarrow M$ is 3-integrable, then tri-secant 3-folds in $M$ are locally parametrized by three functions of one variable.

For 3-integrable GL(2)-structures that arise from PDEs of hydrodynamic type as in Theorem 1.1 (and are therefore also 2-integrable), this parametrization by three functions of one variable confirms the computation presented in [14]. 
Proof. This theorem is proven by applying Cartan-Kähler theory to a differential ideal whose integral manifolds are tri-secant 3-folds $N \subset M$ through an arbitrary tri-secant element $E \in \mathrm{Gr}_{3}\left(\mathbf{T}_{p} M\right)$.

As in the proof of Theorem 3.1, consider a local GL(2) coframe $u$, and let $b=u(p)$. Since $E$ is tri-secant, $b(E)$ is spanned by $\left(g_{1} x-h_{1} y\right)^{4},\left(g_{2} x-\right.$ $\left.h_{2} y\right)^{4}$, and $\left(g_{3} x-h_{3} y\right)^{4}$, distinct, but $u$ can be adapted so that $b(E)$ is spanned by $x^{4}, y^{4}$, and $(x+y)^{4}$. Therefore

$$
\begin{aligned}
b(E)= & \left\{(A+B) x^{4}+B\left(4 x^{3} y+6 x^{2} y^{2}+4 x y^{3}\right)\right. \\
& \left.+(B+C) y^{3}: A, B, C \in \mathbb{R}\right\} \subset \mathcal{V}_{4} .
\end{aligned}
$$

Just as in Theorem 3.1, lift to $\tilde{E}=u_{*}(E) \in \mathrm{Gr}_{2}\left(\mathbf{T}_{b} \mathcal{B}\right)$, which is integral to the linear Pfaffian system $\mathcal{I}$ generated by $\kappa^{-2}=\omega^{-2}-\omega^{0}$ and $\kappa^{2}=\omega^{2}-\omega^{0}$ with the independence condition $\omega^{-4} \wedge \omega^{0} \wedge \omega^{4} \neq 0$. Again, the projection to $M$ of any integral 3 -fold of $\mathcal{I}$ will be a tri-secant 3-fold that passes through $E$, and every tri-secant 3-fold arises this way (up to a GL(2) frame adaptation).

The tableau and torsion of $\mathcal{I}$ are given by

$$
d\left(\begin{array}{c}
\kappa^{-2} \\
\kappa^{2}
\end{array}\right) \equiv\left(\begin{array}{ccc}
\pi_{1} & \pi_{3} & 0 \\
0 & -\pi_{1}-\pi_{2}-\pi_{3} & \pi_{2}
\end{array}\right) \wedge\left(\begin{array}{c}
\omega^{-4} \\
\omega^{0} \\
\omega^{4}
\end{array}\right)+\sum_{a<b}\left(\begin{array}{c}
\tau_{a, b}^{-2} \\
\tau_{a, b}^{2}
\end{array}\right) \omega^{a} \wedge \omega^{b}
$$

modulo $\kappa^{-2}, \kappa^{2}$, where $\pi_{1}=-2 \varphi_{2}, \pi_{2}=2 \varphi_{-2}$ and $\pi_{3}=2 \varphi_{-2}-4 \varphi_{0}+4 \varphi_{2}$. The apparent torsion can be fully absorbed by redefining $\hat{\pi}_{i}=\pi_{i}-p_{i, a} \omega^{a}$ for the parameters

$$
\begin{aligned}
p_{1,4} & =-\tau_{-4,4}^{-2}, \\
p_{2,-4} & =\tau_{-4,4}^{2}, \\
p_{3,4} & =-\tau_{0,4}^{-2}, \\
p_{3,-4} & =-p_{1,-4}-\tau_{-4,4}^{2}-\tau_{-4,0}^{2} \\
p_{2,4} & =-p_{2,0}-p_{3,4}-p_{1,4}+\tau_{0,4}^{2}=-p_{2,0}+\tau_{0,4}^{-2}+\tau_{-4,4}^{-2}+\tau_{0,4}^{2}, \\
p_{1,0} & =p_{3,-4}-\tau_{-4,4}^{-2}=-p_{1,-4}-\tau_{-4,4}^{2}-\tau_{-4,0}^{2}-\tau_{-4,4}^{2} .
\end{aligned}
$$

The integral elements are still free up to arbitrary choice of three functions, $p_{1,-4}, p_{2,0}$ and $p_{3,0}$. Since the Cartan characters are $s_{1}=2, s_{2}=1$ and $s_{3}=$ 0 , but $s_{1}+2 s_{2}+3 s_{3}=4 \neq 3$, the tableau is not involutive; prolongation is required. 
Let $\mathcal{I}^{(1)}$ be the prolonged ideal, which is differentially generated by the forms $\kappa^{-2}$ and $\kappa^{2}$ along with

$$
\begin{aligned}
& \eta^{1}=\pi_{1}+p_{1,-4} \omega^{-4}-\left(p_{1,-4}+\tau_{-4,4}^{2}+\tau_{-4,0}^{2}+\tau_{-4,4}^{2}\right) \omega^{0}-\tau_{-4,4}^{-2} \omega^{4}, \\
& \eta^{2}=\pi_{2}+\tau_{-4,4}^{2} \omega^{-4}+p_{2,0} \omega^{0}+\left(-p_{2,0}+\tau_{0,4}^{-2}+\tau_{-4,4}^{-2}+\tau_{0,4}^{2}\right) \omega^{4}, \text { and } \\
& \eta^{3}=\pi_{3}+\left(-p_{1,-4}-\tau_{-4,4}^{2}-\tau_{-4,0}^{2}\right) \omega^{-4}+p_{3,0} \omega^{0}+-\tau_{0,4}^{-2} \omega^{4} .
\end{aligned}
$$

After this prolongation, the tableau and torsion are given by

$$
d\left(\begin{array}{c}
\kappa^{-2} \\
\kappa^{2} \\
\eta^{1} \\
\eta^{2} \\
\eta^{3}
\end{array}\right) \equiv\left(\begin{array}{ccc}
0 & 0 & 0 \\
0 & 0 & 0 \\
\pi_{4} & -\pi_{4} & 0 \\
0 & \pi_{5} & -\pi_{5} \\
-\pi_{4} & \pi_{6} & 0
\end{array}\right) \wedge\left(\begin{array}{c}
\omega^{-4} \\
\omega^{0} \\
\omega^{4}
\end{array}\right)+\tau^{(1)}(\eta \wedge \eta)
$$

modulo $\kappa^{-2}, \kappa^{2}, \eta^{1}, \eta^{2}, \eta^{3}$. This tableau has Cartan characters $s_{1}=3, s_{2}=0$ and $s_{3}=0$. Applying Cartan's test, $s_{1}+2 s_{2}+3 s_{3}=3$, which matches the dimension of the variety of 3 -dimensional integral elements of $\mathcal{I}^{(1)}$, so the tableau is involutive. When the apparent torsion $\tau^{(1)}$ vanishes or can be absorbed, then in the analytic category Cartan's test for involutivity implies that the integral 3-folds locally depend on three functions of one variable.

In fact, one can say more by analyzing the characteristic variety of the involutive linear Pfaffian system presented in Equation (4.5). Given the highest non-zero Cartan character, $s_{1}=3$, it is clear that the complex characteristic variety has dimension zero and degree three [3, Chapter V]. It is easy to verify that the characteristic variety consists of three real points, so each 3-fold integral to $\mathcal{I}^{(1)}$ is foliated by three families of 2-folds integral to $\mathcal{I}^{(1)}$. A special case of this fact is revisited in Corollary 4.1.

To determine sufficient conditions for existence of tri-secant 3-folds, one must study the unabsorbable portion of the remaining torsion, $\tau^{(1)}$. Because $\tau^{(1)}$ is the torsion of the prolonged system $\mathcal{I}^{(1)}$, it will involve second-order invariants of the $\mathrm{GL}(2)$-structure $\mathcal{B}$ that appear in the Bianchi identity for $\mathcal{B}$, Equation (3.6). Since $T$ is a priori valued in $H^{0,2}(\mathfrak{g l}(2))=\mathcal{V}_{2} \oplus \mathcal{V}_{6} \oplus \mathcal{V}_{8} \oplus$ $\mathcal{V}_{10}$, components of any of the following functions may occur in $\tau^{(1)}$ :

$$
\begin{aligned}
R & : \mathcal{B} \\
r & : \mathcal{B} \rightarrow \mathcal{V}_{2} \otimes\left(\wedge^{2} \mathcal{V}_{4} \otimes\left(\wedge^{2} \mathcal{V}_{4}\right)\right. \\
\nabla T & : \mathcal{B} \rightarrow\left(\mathcal{V}_{2} \oplus \mathcal{V}_{6} \oplus \mathcal{V}_{8} \oplus \mathcal{V}_{10}\right) \otimes \mathcal{V}_{4} \\
Q & : \mathcal{B} \rightarrow \operatorname{Sym}^{2}\left(\mathcal{V}_{2} \oplus \mathcal{V}_{6} \oplus \mathcal{V}_{8} \oplus \mathcal{V}_{10}\right) \cap\left(\mathcal{V}_{4} \otimes \wedge^{3} \mathcal{V}_{4}\right)
\end{aligned}
$$


The vanishing of the unabsorbable portion of $\tau^{(1)}$ will place restrictions on the various irreducible representations appearing in Equation (4.6). The enormous complexity of $Q$ and $\nabla T$ makes decomposition of the unabsorbable portion of $\tau^{(1)}$ extremely difficult. Fortunately, one can make a simplifying assumption that is consistent with the motivating PDE theory in Theorem 1.1; henceforth, all theorems discuss only those GL(2)-structures that are both 2-integrable and 3-integrable.

Corollary 4.1. If $\mathcal{B} \rightarrow M$ is a 2,3-integrable $\mathrm{GL}(2)$-structure, then any tri-secant $N^{3} \subset M$ is triply foliated by bi-secant surfaces. Moreover, the net defined by $\mathbf{T} N \cap \mathbf{C}$ is a coordinate net.

The triple foliation by bi-secant surfaces appears to be new, though it is not surprising based on the description of highest-weight polynomial subspaces of $\mathcal{V}_{n}$ seen in [13]. This foliation shows that the "holonomic characteristic net" condition for tri-secant 3-folds seen in [14] is superfluous since 2-integrability is implied for GL(2)-structures arising from Hessian hydrodynamic PDEs.

Proof. Without loss of generality in a contractible neighborhood in $M$, one may apply a GL(2) change-of-frame so that $\mathbf{T} N=\left\{A x^{4}+B(x+y)^{4}+\right.$ $\left.C y^{4}\right\}$. The characteristic net, $\mathbf{T} N \cap \mathbf{C}$, is given by its tangent planes

$$
\begin{aligned}
& K_{1}=\operatorname{span}\left\{(x+y)^{4}, y^{4}\right\}, \\
& K_{2}=\operatorname{span}\left\{x^{4}, y^{4}\right\}, \text { and } \\
& K_{3}=\operatorname{span}\left\{x^{4},(x+y)^{4}\right\} .
\end{aligned}
$$

Each of $K_{1}, K_{2}$ and $K_{3}$ is clearly bi-secant. It suffices to prove that they are everywhere tangent to the level sets of a coordinate system on $N$, as these level sets will be bi-secant surfaces.

Recall that $N$ is defined by the projection of an integral manifold of the prolonged system $\mathcal{I}^{(1)}$ on $\mathcal{B} \times \mathbb{R}^{3}$ from Theorem 4.1. In particular, there is a submanifold $\tilde{N} \subset \mathcal{B}$ with embedding map $\tilde{\nu}: \tilde{N} \rightarrow \mathcal{B}$ depending on three parameters $P_{1}, P_{2}, P_{3}$ such that each $\mathbf{T}_{p} N$ is the projection of

$$
\begin{aligned}
\mathbf{T}_{b} \tilde{N}=\operatorname{ker}( & \left\{\kappa^{-2}, \kappa^{2}, \eta^{1}, \eta^{2}, \eta^{3},\right. \\
& \pi_{4}-P_{1}\left(\omega^{-4}-\omega^{0}\right), \\
& \pi_{5}-P_{2}\left(\omega^{0}-\omega^{4}\right), \\
& \left.\left.\pi_{6}-P_{1} \omega^{-4}+P_{3} \omega^{0}\right\}\right) .
\end{aligned}
$$


Let $\Upsilon^{1}=\omega^{-4}-\omega^{0}, \Upsilon^{2}=\omega^{0}$ and $\Upsilon^{3}=\omega^{4}-\omega^{0}$. Therefore, at each basepoint, $K_{i} \subset \mathbf{T}_{p} N$ is the projection of the plane $\operatorname{ker}\left(\tilde{\nu}^{*}\left(\Upsilon^{i}\right)\right) \subset \mathbf{T}_{b} \tilde{N}$. One can easily compute that $d\left(\tilde{\nu}^{*}\left(\Upsilon^{i}\right)\right) \equiv 0$ modulo $\tilde{\nu}^{*}\left(\Upsilon^{i}\right)$ for each $i$; therefore, the Frobenius theorem provides local coordinates $\left(s^{1}, s^{2}, s^{3}\right)$ on $N$ such that $K_{i}=\operatorname{ker}\left(d s^{i}\right)$.

When $\mathcal{B}$ arises from a Hessian hydrodynamic PDE, these coordinates are called the Riemann invariants of the hydrodynamic reduction.

Theorem 4.2 (2,3-integrable GL(2)-structure equations). $A$ GL(2)structure $\mathcal{B}$ is 2,3-integrable if and only if the torsion $T$ of $\mathcal{B}$ only takes values in $\mathcal{V}_{8}$ and the curvature is a function of $T$. In particular, every 2,3integrable $\mathrm{GL}(2)$-structure $\mathcal{B}$ has the following structure equations:

$$
\begin{aligned}
d \omega= & -\langle\varphi, \omega\rangle_{1}-\langle\lambda, \omega\rangle_{0}+\left\langle T,\langle\omega, \omega\rangle_{1}\right\rangle_{5}, \\
d \lambda= & 0 \\
d \varphi= & -\frac{1}{2}\langle\varphi, \varphi\rangle_{1}-2080\left\langle\langle T, T\rangle_{8},\langle\omega, \omega\rangle_{3}\right\rangle_{0}+64\left\langle\langle T, T\rangle_{6},\langle\omega, \omega\rangle_{3}\right\rangle_{2} \\
& -\frac{88}{7}\left\langle\langle T, T\rangle_{6},\langle\omega, \omega\rangle_{1}\right\rangle_{4}+\frac{24}{7}\left\langle\langle T, T\rangle_{4},\langle\omega, \omega\rangle_{1}\right\rangle_{6}, \\
d T= & J(T)\left(\begin{array}{c}
\omega \\
\lambda \\
\varphi
\end{array}\right)
\end{aligned}
$$

for a $9 \times 9$ matrix-valued function $J$ whose entries are linear and quadratic polynomials in the coefficients of $T$, as provided in Appendix $A$ and [26, 27].

Proof. 2-integrability implies that $T=T_{8}$, so $R, r, \nabla(T)$ and $Q$ decompose as in Equation (3.8) with the relations implied in Theorem 3.1. 3integrability implies the vanishing of the GL(2) orbit of the unabsorbable portion of $\tau^{(1)}$ in Equation (4.5). The vanishing of $\tau^{(1)}$ and the Bianchi identity $\left(d^{2}=0\right)$ together imply the additional relations $S_{12}=0,7 S_{8}=24 Q_{8}=$ $24\langle T, T\rangle_{4}, S_{6}=0,21 S_{4}=8 Q_{4}=8\langle T, T\rangle_{6}$ and $R_{0}^{2}=-2080\langle T, T\rangle_{8}$.

Therefore, the curvatures, $R$ and $r$, are quadratic functions of $T$. The derivative of torsion, $d T$, is also a quadratic function of $T$, as expressed in the matrix $J(T)$. Hence, $T$ is the only invariant of any order for 2,3-integrable $\mathrm{GL}(2)$-structures.

Definition 4.1. The notation $(\mathcal{B}, M, p)_{2,3}$ indicates a smooth 2,3-integrable $\mathrm{GL}(2)$-structure $\pi: \mathcal{B} \rightarrow M$ such that $p \in M$ and such that $M$ is connected. 
The condition that $M$ is connected is crucial in what follows, and pointedness is technically useful.

Definition 4.2 (Representatives). $(\mathcal{B}, M, p)_{2,3}$ represents $v \in \mathcal{V}_{8}$ if $v \in T\left(\mathcal{B}_{p}\right)$. More generally, a 2,3-integrable $\mathrm{GL}(2)$-structure $\mathcal{B} \rightarrow M$ represents $v \in \mathcal{V}_{8}$ if $T(\mathcal{B}) \ni v$. Likewise, a Hessian hydrodynamic PDE represents $v \in \mathcal{V}_{8}$ if the induced 2,3-integrable GL(2)-structure over $M=F^{-1}(0)$ represents $v$.

Lemma 4.1. The singular distribution on $\mathcal{V}_{8}$ defined by the columns of the matrix $J$ is integrable, providing a stratification of $\mathcal{V}_{8}$ into leaves that are submanifolds. That is, for any $v \in \mathcal{V}_{8}$, there exists a unique submanifold $\mathcal{O}_{J}(v)$ such that $\mathbf{T}_{v} \mathcal{O}_{J}(v)=$ range $J(v)$.

Lemma 4.1 is elementary in its modern interpretation using the theory of Lie algebroids and smooth groupoids. Since the equations in Theorem 4.2 are closed under exterior derivative, the matrix $J$ is closed under the corresponding bracket, so it defines the anchor map of a Lie algebroid over $\mathcal{V}_{8}$. This Lie algebroid is neither regular nor transitive, but there exists an integrating groupoid over the base, $\mathcal{V}_{8}$. This groupoid is smooth, but it may not be a Lie groupoid. However, the groupoid is transitive when restricted to each of its orbits, $\mathcal{O}_{J}(v)$, and each orbit is a submanifold of the base [12, 23, 28]. These orbits may be regarded as the leaves of a singular foliation of $\mathcal{V}_{8}$ such that $\mathbf{T}_{v} \mathcal{O}_{J}(v)$ is spanned by the columns of $J(v)$.

Corollary 4.2. For any $v \in \mathcal{V}_{8}$, there exists a real-analytic connected 2,3integrable $\mathrm{GL}(2)$-structure $\mathcal{B} \rightarrow M$ such that $T(\mathcal{B}) \ni v$. That is, every $v \in \mathcal{V}_{8}$ is represented by some real-analytic $(\mathcal{B}, M, p)_{2,3}$. Moreover, $J$ has constant rank on any such $\mathcal{B}$.

Proof. Since the structure equations in Theorem 4.2 are closed under exterior derivative, this is a direct application of the existence part of Cartan's structure theorem, which is an generalization of Lie's third fundamental theorem to the intransitive case [8]. See [6, Appendix A] for a clear summary of the special case of Cartan's structure theorem needed here. As used here, Cartan's structure theorem holds in the smooth category; however, because $T \cdot T$ and $J(T)$ are real-analytic (in fact, linear and quadratic), one can use the Cartan-Kähler machinery to produce a local real-analytic solution $\mathcal{B}$ of the structure equations. On any solution $\mathcal{B}$, the image of $\left.d T\right|_{b}$ is the image of the columns of the matrix $J(T(b))$, and these columns span the tangent 
space of the submanifold $\mathcal{O}_{J}(T(b))$. Thus, on any connected $\mathcal{B}$ containing $b$, the rank of $J$ equals the dimension of $\mathcal{O}_{J}(T(b))$.

Corollary 4.3. $(\mathcal{B}, M, p)_{2,3}$ and $(\hat{\mathcal{B}}, \hat{M}, \hat{p})_{2,3}$ admit a local $\mathrm{GL}(2)$ equivalence $f: M \rightarrow \hat{M}$ with $f(p)=\hat{p}$ if and only if $T\left(\mathcal{B}_{p}\right) \cap \hat{T}\left(\hat{\mathcal{B}}_{\hat{p}}\right) \neq \emptyset$. That is, the value $T(b)$ uniquely defines $(\mathcal{B}, M, p)_{2,3}$ in a neighborhood of $p=\pi(b)$, and this local $\mathrm{GL}(2)$-structure is real-analytic.

Proof. This is a direct application of the local uniqueness part of Cartan's structure theorem. Again, the theorem applies here in the smooth category, but the real-analyticity of the structure equations implies that any 2,3integrable GL(2)-structure is locally equivalent to the real-analytic GL(2)structure produced in Corollary 4.2 .

Corollaries 4.2 and 4.3 establish the primacy of $T$ in the study 2,3integrable GL(2)-structures and the related PDEs. However, Corollary 4.3 provides only local GL(2)-equivalence, so it is useful to have a weaker "chainwise" notion of equivalence that applies to global (but still connected) GL(2)structures.

Definition 4.3 (Leaf-equivalence). $\left(\mathcal{B}_{0}, M_{0}, p_{0}\right)_{2,3}$ and $\left(\mathcal{B}_{k}, M_{k}, p_{k}\right)_{2,3}$ are said to be leaf-equivalent if there exist finite sequences $\left\{\left(\mathcal{B}_{i}, M_{i}, p_{i}\right)_{2,3}\right\}$ and $\left\{v_{i}\right\}$ with $1 \leq i \leq k-1$ such that $\left(\mathcal{B}_{i}, M_{i}, p_{i}\right)_{2,3}$ and $\left(\mathcal{B}_{i+1}, M_{i+1}, p_{i+1}\right)_{2,3}$ both represent $v_{i}$ for $0 \leq i \leq k-1$.

The term "leaf-equivalence" arises from the leaves of the singular foliation of $\mathcal{V}_{8}$ from Lemma 4.1. These leaves separate all possible $(\mathcal{B}, M, p)_{2,3}$ 's into equivalence classes by the value of $T$. By slight abuse of notation, if $(\mathcal{B}, M, p)_{2,3}$ represents $v$, then write $\mathcal{O}_{J}(\mathcal{B})$ to denote $\mathcal{O}_{J}(v)$. Leaf-equivalence can now be rewritten more succinctly.

Theorem 4.3 (Leaf-equivalence). $(\mathcal{B}, M, p)_{2,3}$ and $(\hat{\mathcal{B}}, \hat{M}, \hat{p})_{2,3}$ are leafequivalent if and only if $\mathcal{O}_{J}(\mathcal{B})=\mathcal{O}_{J}(\hat{\mathcal{B}})$. Moreover, $T: \mathcal{B} \rightarrow \mathcal{O}_{J}(\mathcal{B})$ is a submersion.

Although $T: \mathcal{B} \rightarrow \mathcal{O}_{J}(\mathcal{B})$ is an open map, it need not be surjective.

\section{The classification}

To classify connected 2,3-integrable GL(2)-structures is to explicitly identify the leaves of the foliation of $\mathcal{V}_{8}$. To identify the leaves of the foliation is to 
identify connected GL(2)-invariant submanifolds of $\mathcal{V}_{8}$ where the rank of $J(v)$ is constant. This is all ultimately achieved in Theorem 5.1 thanks to the observations in Lemmas 5.1 and 5.2.

Lemma 5.1. In vector space of 14 th degree polynomials in the variables $v_{-8}, \ldots, v_{8}$, the determinant of $J(v)$ is a non-zero scalar multiple of the discriminant of the polynomial $v \in \mathcal{V}_{8}$. Therefore, $J(v)$ is non-singular if and only if $v$ has eight distinct roots. Moreover, if $v$ is a non-trivial polynomial with $k$ distinct roots, then the rank of $J(v)$ is $k+1$.

Proof. The determinant statement is verified by computing the g.c.d. of the two polynomials, $\operatorname{det} J(v)$ and $\operatorname{disc}(v)$. The rank statement is directly verified by writing $v=\left(g_{1} x-h_{1} y\right)\left(g_{2} x-h_{2} y\right) \cdots\left(g_{8} x-h_{8} y\right)$, imposing multiplicity on the $h_{i}$ 's and $g_{i}$ 's and computing the rank of $J(v)$ directly.

As it happens, a simple exercise in linear fractional transformations shows that the multiplicity of the roots is preserved by the GL(2) action on $\mathcal{V}_{8}[1,11]$.

Lemma 5.2. For $v(x, y) \in \mathcal{V}_{8}$, the multiplicity and complex type of the roots are preserved under the irreducible action of $\mathrm{GL}(2, \mathbb{R})$.

Bearing in mind this lemma, notational shorthand for the root type of a polynomial $v(x, y) \in \mathcal{V}_{8}$ is useful: Suppose $v(x, y)$ factors as $\left(g_{1} x-\right.$ $\left.h_{1} y\right)^{r_{1}} \cdots\left(g_{m} x-h_{m} y\right)^{r_{m}}$ such that $r_{1} \geq r_{2} \geq \cdots \geq r_{m}$ and $r_{1}+r_{2}+\cdots+$ $r_{m}=8$. These exponents define a partition of 8 that is written as $\left\{r_{1}, r_{2}, \ldots, r_{m}\right\}$. If the roots $g_{k} / h_{k}$ and $g_{k+1} / h_{k+1}$ are complex conjugates, then denote this by enclosing their exponents in square-braces: $\left\{r_{1}, \ldots,\left[r_{k}, r_{k+1}\right], \ldots, r_{m}\right\}$. Denote the root type containing $v(x, y)$ by $[v(x, y)]$. A root type is equivalent to the subset of $\mathcal{V}_{8}$ comprised of all polynomials that factor according to the given partition and complex-conjugate pairing. For example, $\left[x^{4}(x+i y)^{2}(x-i y)^{2}\right]=\left[(x-5 y)^{4}(x+2 i y)^{2}(x-\right.$ $\left.2 i y)^{2}\right]=\{4,[2,2]\} \subset \mathcal{V}_{8}$. Let $\{0\}$ denote the trivial root type, the zero polynomial. There are 54 non-trivial root types that partition $\mathcal{V}_{8}$ into strata from dimension two to dimension nine, as represented in figure 1.

In figure 1, arrows mean "closure contains." Shaded nodes represent root types that contain exactly one GL(2) orbit. Oval nodes represent open root types. The square node represents the nearly closed root type, $\{8\}$, which (when 0 is included) is the rational normal cone in $\mathcal{V}_{8}$. Hexagonal nodes represent root classes that are neither closed nor open. Note that strictly real root types actually have two connected components (for example, $\{8\}$ is 


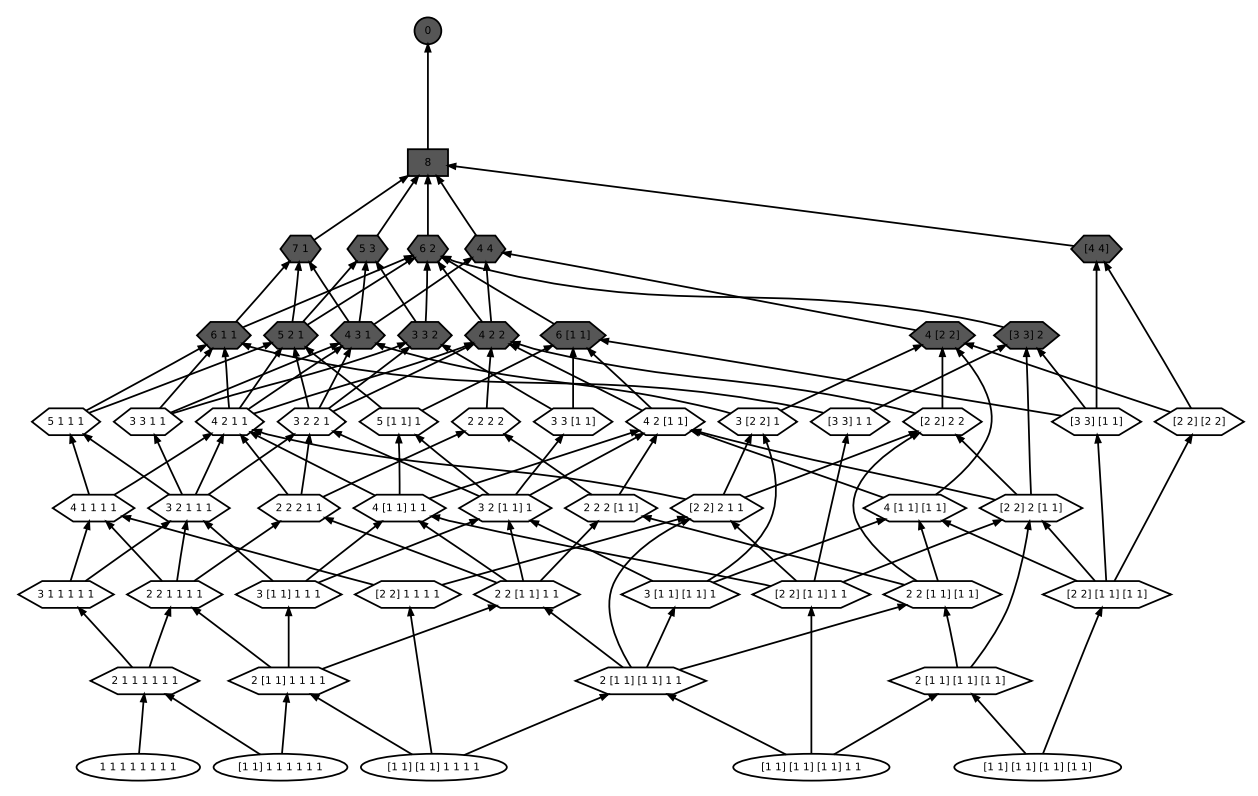

Figure 1: The stratification of $\mathcal{V}_{8}$ into the 55 root types, sorted by dimension. Shaded nodes indicate root types that contain exactly one GL(2) orbit. The square node is closed (if 0 is included); the oval nodes are open, and the hexagonal nodes are neither closed nor open.

comprised of the two ends of the rational normal cone), but this is another artifact of the projective nature of the group action, as the two ends are in the same GL(2) orbit by the $-I_{9}$ action.

Theorem 5.1 (Leaf-equivalence classes). The leaf-equivalence classes of connected 2,3-integrable $\mathrm{GL}(2)$-structures are exactly the root types in $\mathcal{V}_{8}$. That is, for all $v \in \mathcal{V}_{8}, \mathcal{O}_{J}(v)=[v]$.

Proof. For each $v$, both $\mathcal{O}_{J}(v)$ and $[v]$ are smooth submanifolds of $\mathcal{V}_{8}$, and by Lemma 5.1 they have the same dimension. To prove that $\mathcal{O}_{J}(v)=[v]$, it suffices to prove that $\mathbf{T}_{v} \mathcal{O}_{J}(v)=\mathbf{T}_{v}[v]$ for all $v \in \mathcal{V}_{8}$.

Fix a root type $[v]$ and an arbitrary $v \in[v]$. Note that

$$
\mathbf{T}_{v} \mathcal{O}_{J}(\mathcal{B})=D T_{b}\left(\mathbf{T}_{b} \mathcal{B}\right)=\text { range } J(v) .
$$

Thus it suffices to find, for each column $J_{i}(v)$, a tangent vector $D(v) \in \mathbf{T}_{v}[v]$ such that $D(v)=J_{i}(v)$. As it happens, the equations defining the various $D(v)$ are easily solvable at arbitrary points in all 54 non-trivial root types 
and for all columns of $J$. Because both the leaf-equivalence classes and the root types partition $\mathcal{V}_{8}$ by smooth submanifolds, and because $\mathbf{T}_{v} \mathcal{O}_{J}(v)=$ $\mathbf{T}_{v}[v]$ for all $v \in \mathcal{V}_{8}$, the partitions must be identical.

To illustrate the computations, consider an arbitrary point $v=(g x+$ $h y)^{8}$ in the root type $[v]=\{8\}$. An arbitrary element of $\mathbf{T}_{v}[v]$ looks like

$$
D(v)=8(G x+H y)(g x+h y)^{7}=\left(\begin{array}{c}
8 G g^{7} \\
H g^{7}+7 G h g^{6} \\
2 H h g^{6}+6 G h^{2} g^{5} \\
3 H h^{2} g^{5}+5 G g^{4} h^{3} \\
4 G g^{3} h^{4}+4 H g^{4} h^{3} \\
5 H g^{3} h^{4}+3 G h^{5} g^{2} \\
6 H h^{5} g^{2}+2 G h^{6} g \\
7 H h^{6} g+G h^{7} \\
8 H h^{7}
\end{array}\right)
$$

Therefore, one must solve $D(v)=J_{i}(v)$ for $G$ and $H$ in each of the columns $i=1, \ldots, 9$. In this case,

$$
J(v)=\left(\begin{array}{ccccccccc}
0 & 0 & 0 & 0 & 0 & g^{8} & -16 h g^{7} & 16 g^{8} & 0 \\
0 & 0 & 0 & 0 & 0 & h g^{7} & -14 h^{2} g^{6} & 12 h g^{7} & 2 g^{8} \\
0 & 0 & 0 & 0 & 0 & h^{2} g^{6} & -12 h^{3} g^{5} & 8 h^{2} g^{6} & 4 h g^{7} \\
0 & 0 & 0 & 0 & 0 & h^{3} g^{5} & -10 h^{4} g^{4} & 4 h^{3} g^{5} & 6 h^{2} g^{6} \\
0 & 0 & 0 & 0 & 0 & h^{4} g^{4} & -8 h^{5} g^{3} & 0 & 8 h^{3} g^{5} \\
0 & 0 & 0 & 0 & 0 & h^{5} g^{3} & -6 h^{6} g^{2} & -4 h^{5} g^{3} & 10 h^{4} g^{4} \\
0 & 0 & 0 & 0 & 0 & h^{6} g^{2} & -4 h^{7} g & -8 h^{6} g^{2} & 12 h^{5} g^{3} \\
0 & 0 & 0 & 0 & 0 & h^{7} g & -2 h^{8} & -12 h^{7} g & 14 h^{6} g^{2} \\
0 & 0 & 0 & 0 & 0 & h^{8} & 0 & -16 h^{8} & 16 h^{7} g
\end{array}\right) .
$$

For each column $J_{i}(v)$, it is easy to see the solution values of $G$ and $H$.

For the other root types, the computations are similar but somewhat more complicated. All that matters is the fact that they can be solved for arbitrary $v$.

\subsection{Symmetry reduction}

Geometrically, it is interesting to reduce all symmetry from a structure. Fix $(\mathcal{B}, M, p)_{2,3}$ and $v \in T(\mathcal{B})$. Let $\mathcal{B}^{v}=\{b \in \mathcal{B}: T(b)=v\}$. This is a sub-bundle of $\mathcal{B}$ with fiber group $\operatorname{Stab}(v) \subset G L(2)$. If $\operatorname{dim}[v] \leq 3$, then the stabilizer group is smooth; however, the stabilizer groups must be discrete for all 
the larger root types. The stabilizer group, either smooth or discrete, must always appear in the well-known list of $\mathrm{GL}(2, \mathbb{C})$-stabilizers of polynomials [2]. In the discrete case, the fiber must therefore be either a cyclic group or a dihedral group [27].

The structure equations for $\mathcal{B}^{v}$ show no dependence on $T$ (as it has been fixed), so a neighborhood in $\mathcal{B}^{v}$ is a local Lie group of dimension $9-\operatorname{dim}[v]$, and $M$ is locally the homogeneous space $\mathcal{B}^{v} / \operatorname{Stab}(v)$. The relations defined by $\operatorname{ker} d T(v)=\operatorname{ker} J(v)$ determine the pull-backs of $\omega, \lambda$, and $\varphi$ to $\mathcal{B}^{v}$, so one may explicitly reduce Equation (4.9) to obtain structure equations for the local Lie group $\mathcal{B}^{v}$.

If $v=0$, then $\operatorname{Stab}(v)=\mathrm{GL}(2)$, so the local flat 2,3-integrable GL(2)structure is the local Lie group of dimension 9 obtained by setting $T=0$ in Equation (4.9).

The root type $\{8\}$ is a single $\mathrm{GL}(2)$ orbit, so any representative will generate all representatives. Suppose $v=x^{8}$. Then $d T_{-8}\left(x^{8}\right)=\lambda+16 \varphi_{0}$ and $d T_{-6}\left(x^{8}\right)=2 \varphi_{2}$ while $d T_{k}=0$ for $k>-6$. Since $d T$ is a vertical 1 -form on $\mathcal{B}$, the value of $T$ only varies in the fiber of $\mathcal{B}$. The reduced structure equations are

$$
\begin{aligned}
d \omega^{-4} & =24 \varphi_{0} \wedge \omega^{-4}-8 \varphi_{-2} \wedge \omega^{-2}+2 \times 322,560 \omega^{0} \wedge \omega^{4}, \\
d \omega^{-2} & =20 \varphi_{0} \wedge \omega^{-2}-6 \varphi_{-2} \wedge \omega^{0}+322,560 \omega^{2} \wedge \omega^{4}, \\
d \omega^{0} & =16 \varphi_{0} \wedge \omega^{0}-4 \varphi_{-2} \wedge \omega^{2} \\
d \omega^{2} & =12 \varphi_{0} \wedge \omega^{2}-2 \varphi_{-2} \wedge \omega^{4} \\
d \omega^{4} & =8 \varphi_{0} \wedge \omega^{4} \\
d \varphi_{0} & =0, \quad d \varphi_{-2}=4 \varphi_{0} \wedge \varphi_{-2} .
\end{aligned}
$$

These equations can be easily integrated, so $\mathcal{B}^{x^{8}}$ has coordinates $\xi^{-4}, \xi^{-2}$, $\xi^{0}, \xi^{2}, \xi^{4}, a$ and $b$ such that

$$
\begin{aligned}
\varphi_{0}= & a^{-1} d a \\
\varphi_{-2}= & a^{4} d b \\
\omega^{4}= & a^{8} d \xi^{4}, \\
\omega^{2}= & a^{12}\left(d \xi^{2}-2 b d \xi^{4}\right), \\
\omega^{0}= & a^{16}\left(d \xi^{0}-4 b d \xi^{2}+4 b^{2} d \xi^{4}\right), \\
\omega^{-2}= & a^{20}\left(d \xi^{-2}-6 b d \xi^{0}+12 b^{2} d \xi^{2}-8 b^{3} d \xi^{4}-322,560 \xi^{4} d \xi^{2}\right), \\
\omega^{-4}= & a^{24}\left(d \xi^{-4}-8 b d \xi^{-2}+24 b^{2} d \xi^{0}-32 b^{3} d \xi^{2}+16 b^{4} d \xi^{4}\right. \\
& \left.+8(322,560) \xi^{4} b d \xi^{2}-2(322,560) \xi^{4} d \xi^{0}\right) .
\end{aligned}
$$


The field of rational normal cones can now be written in these local coordinates, since $\mathbf{C}_{p}=\left\{\left\langle u_{p}(v), u_{p}(v)\right\rangle_{2}: v \in \mathbf{T}_{p} M\right\}$ for a local section $u$ of $\mathcal{B}$.

Each of the 3-dimensional root types is a single GL(2)-orbit. Again, $T(\mathcal{B})=[v]$ for any $v$ in the root type, so an arbitrary representative $v$ may be chosen for any $(\mathcal{B}, M, p)_{2,3}$. Each $v$ has a 1-dimensional stabilizer, which is a Lie subgroup of GL(2). The corresponding Lie algebras are easy to compute $[2,27]$. In all cases, $d T$ has both vertical and semi-basic components, so the embedding of the stabilizer fiber group varies over $M$. The reduced structure $\mathcal{B}^{v}$ is a 6 -dimensional Lie group.

If $\operatorname{dim}[v]=4$, then $\mathcal{B}^{v}$ is a finite cover of $M$, but there are only eight such structures, since each root type with three roots is a single GL(2)orbit. This contrasts with the case $\operatorname{dim}[v] \geq 5$. If $\operatorname{dim}[v] \geq 5$, then $\mathcal{B}^{v}$ is a finite cover of $M$, but there is no reason to believe that $T(\mathcal{B}) \subset[v]$ implies $T(\mathcal{B})=[v]$ when $\operatorname{dim}[v] \geq 5$. Consider $[v]=\{2,2,2,2\}$. The quotient space $\{2,2,2,2\} / \mathrm{GL}(2)$ has orbifold singularities; for example, the point $x^{2} y^{2}(x+$ $y)^{2}(x-y)^{2}$ has an eight-element stabilizer group, but all nearby points have trivial stabilizer groups. The existence of these orbifold singularities implies that there cannot be a surjective smooth map $M \rightarrow[v] / \mathrm{GL}(2)$. If $T(\mathcal{B})$ is a proper subset of $[v]$ for all $\mathcal{B}$ representing $v$, then by Theorem 5.1 a finite sequence of 2,3-integrable GL(2)-structures connects any two points in the leaf, but infinitely many locally distinct GL(2)-structures are required to cover the entire leaf. This behavior is closely related to the topology of $\mathbb{C P}^{n}$ with $k$ marked points, up to $\operatorname{GL}(2, \mathbb{C})$ action, which is a difficult and wellknown problem in complex algebraic geometry. The real case encountered here is both harder and less well-known than the classical complex case. The most relevant result is [10], which shows that the orbifold singularities present in the larger leaves of $\mathcal{V}_{8}$ are so bad that they preclude the existence of Riemannian metrics on the leaves.

In the open case, $\operatorname{dim}[v]=9$, so $J(T)$ has maximum rank, and $T$ itself provides local coordinates on $\mathcal{B}$.

This discussion is summarized by the shape of the nodes in figure 2 .

\section{GL(2) PDEs}

This section contains various conclusions regarding Hessian hydrodynamic PDEs that can be inferred from Theorem 1.1 and Theorem 5.1. Recall that a PDE $F=0$ is said to have $k$ symmetries if the Lie algebra of point symmetries of $F^{-1}(0)$ has dimension $k$. 
Lemma 6.1. A Hessian hydrodynamic PDE $F\left(u_{i j}\right)=0$ representing $v \in$ $\mathcal{V}_{8}$ has $k$ symmetries if and only if $\operatorname{dim}[v]=9-k$.

Proof. The contact transformations by $C S p(3)$ induce the local automorphisms on $\mathcal{B}$, but an automorphism $\psi: \mathcal{B} \rightarrow \mathcal{B}$ near $b$ is a symmetry if and only if the structure equations are preserved by $\psi^{*}$. This can happen if and only if $T(b)$ is preserved near $\psi(b)$. In particular, $\psi^{*}(d T)=d T$ if and only if $\psi^{*}$ is the identity on the range of $d T$, which has dimension $\operatorname{dim}[v]$.

Corollary 6.1. There is no Hessian hydrodynamic PDE with exactly eight symmetries.

Proof. There is no root type of dimension one.

The classification provides even more bountiful information about the Hessian hydrodynamic PDEs, because Theorem 4.2 allows a converse of Theorem 1.1.

Theorem 6.1. Every $(\mathcal{B}, M, p)_{2,3}$ is realized, locally near $p$, by a Hessian hydrodynamic PDE.

Proof. The structure $(\mathcal{B}, M, p)_{2,3}$ is realized, locally near $p$, by a Hessian hydrodynamic PDE as in Lemma 1.2 if and only if there exists a neighborhood $M^{\prime} \subset M$ of $p$ and an embedding $i: M^{\prime} \rightarrow \Lambda^{o}$ such that the distribution of rational normal cones $i_{*}\left(\mathbf{C}\left(M^{\prime}\right)\right)$ over $i\left(M^{\prime}\right)$ is the same as the intersection of $i_{*}\left(\mathbf{T} M^{\prime}\right)$ with the distribution of Veronese cones over $\Lambda^{o}$.

Since the fibers $\mathcal{B}$ are exactly the symmetries of $\mathbf{C}$ and the fibers of $C S p(3)^{\circ}$ are exactly the symmetries of the distribution of Veronese cones, it suffices to establish a bundle immersion $h: \mathcal{B}\left(M^{\prime}\right) \rightarrow C S p(3)^{o}$ covering an embedding $i: M^{\prime} \rightarrow \Lambda^{o}$.

Let $\mu$ denote the Maurer-Cartan form of $C S p(3)$, which is of the form

$$
\mu=\left(\begin{array}{cc}
\beta & \gamma \\
\alpha & -\beta^{t}
\end{array}\right), \quad \alpha=\alpha^{t}, \quad \gamma=\gamma^{t}, \quad d \mu+\mu \wedge \mu=0
$$

(One may assume that the conformal scaling has been incorporated into $\beta$, as it is below.) By Equations (1.13) and (1.14), the components $\beta$ and $\gamma$ are vertical for $\Pi$, and $\alpha$ is semi-basic.

Recall the Fundamental Lemma of Lie Groups [22, Theorem 1.6.10]: If there exists $\eta: \mathbf{T B} \rightarrow \mathfrak{c s p}(3)$ such that $d \eta+\eta \wedge \eta=0$, then for any $b \in \mathcal{B}$, there exists a neighborhood $\mathcal{B}^{\prime}$ of $b$ and a map $h: \mathcal{B}^{\prime} \rightarrow C S p(3)$ such that 
$h^{*}(\mu)=\eta$. Moreover, if $h^{*}(\alpha)$ is semi-basic, then the fibers of $\mathcal{B}^{\prime}$ immerse into the fibers of $C S p(3)$.

Therefore, it suffices to construct a $\mathfrak{c} \mathfrak{s p}(3)$-valued Maurer-Cartan form $\eta$ on $\mathcal{B}$ such that the entries of the lower-left symmetric submatrix of $\eta$, namely $h^{*}\left(\alpha_{i j}\right)$, are semi-basic on $\mathcal{B}$. For brevity, the pull-backs $h^{*}(\cdot)$ are dropped from the notation henceforth.

The condition that $\alpha$ is semi-basic is that $\alpha_{i j}=A_{i j a} \omega^{a}$. Note that $\alpha$ : $\mathcal{V}_{4} \rightarrow \operatorname{Sym}^{2}\left(\mathbb{R}^{3}\right)=\operatorname{Sym}^{2}\left(\mathcal{V}_{2}\right)$, and recall that for $u, v \in \mathcal{V}_{2}$ the symmetric tensor is given by

$$
\begin{aligned}
& \left(\begin{array}{c}
u^{-2} \\
u^{0} \\
v^{2}
\end{array}\right) \circ\left(\begin{array}{c}
v^{-2} \\
v^{0} \\
v^{2}
\end{array}\right) \\
& \quad=\frac{1}{2}\left(\begin{array}{ccc}
u^{-2} v^{-2}+v^{-2} u^{-2} & u^{-2} v^{0}+v^{-2} u^{0} & u^{-2} v^{2}+v^{-2} u^{2} \\
u^{0} v^{-2}+v^{0} u^{-2} & u^{0} v^{0}+v^{0} u^{0} & u^{0} v^{2}+v^{0} u^{2} \\
u^{2} v^{-2}+v^{2} u^{-2} & u^{2} v^{0}+v^{2} u^{0} & u^{2} v^{2}+v^{2} u^{2}
\end{array}\right)
\end{aligned}
$$

Therefore, to respect the weights in the GL(2) representation, $\alpha$ must have the following form for constants $A_{-4}, A_{-2}, A_{0}, A_{0}^{\prime}, A_{2}$, and $A_{4}$ :

$$
\alpha=\left(\begin{array}{ccccc}
A_{-4} \omega^{-4} & A_{-2} \omega^{-2} & A_{0} \omega^{0} \\
A_{-2} \omega^{-2} & A_{0}^{\prime} \omega^{0} & A_{2} \omega^{2} \\
A_{0} \omega^{0} & A_{0} \omega^{2} & A_{4} \omega^{4}
\end{array}\right)
$$

Writing $\beta=\beta_{\varphi}(\varphi)+\beta_{\lambda}(\lambda)+\beta_{T}(\omega)$, it is apparent that $\beta_{\varphi}$ must be the representation $\mathfrak{s l}(2) \rightarrow M_{3 \times 3}(\mathbb{R})$ such that the natural action of $M_{3 \times 3}(\mathbb{R})$ on $\operatorname{Sym}^{2}\left(\mathcal{V}_{2}\right)$ is induced by the natural action of $\mathfrak{s l}(2)$ on $\omega \in \mathcal{V}_{4}$. One can now easily verify the following formulas:

$$
\begin{aligned}
\alpha & =\left(\begin{array}{ccc}
\omega^{-4} & \omega^{-2} & \omega^{0} \\
\omega^{-2} & \omega^{0} & \omega^{2} \\
\omega^{0} & \omega^{2} & \omega^{4}
\end{array}\right), \\
\beta & =\left(\begin{array}{ccc}
4 \varphi_{0} & 2 \varphi_{2} & 0 \\
-4 \varphi_{-2} & 0 & 4 \varphi_{2} \\
0 & -2 \varphi_{-2} & -4 \varphi_{0}
\end{array}\right)-\frac{1}{2} \lambda I_{3}+\beta_{T}(\omega) .
\end{aligned}
$$

Here, the $-\frac{1}{2} \lambda I_{3}$ component of $\beta$ is simply the scaling action of GL(2) as represented by the scaling action in $C S p(3)$. If $\mathrm{PGL}(2)$ and $\mathrm{Sp}(3)$ were used instead, it would not appear. 
All that remains is to find $\beta_{T}$ and $\gamma$ such that $d \eta+\eta \wedge \eta=0$. This is arithmetic, and solutions exist. The simplest $\eta$ (the one where the undetermined coefficients in $\beta_{T}$ are set to 0 ) is provided in [26].

Theorem 6.1 allows explicit construction of several of the mostsymmetric Hessian hydrodynamic PDEs. Each of the root types with three or fewer roots is itself closed under GL(2); hence, the torsion of any GL(2)structure covers the entire root type. This allows a first step at constructing all Hessian hydrodynamic PDEs. However, Theorem 6.1 does not say that there are only 55 Hessian hydrodynamic PDEs up to $C S p(3)$ actions. There is no reason to believe that the torsion map $T: \mathcal{B} \rightarrow \mathcal{V}_{8}$ is surjective for the larger root types. When $T$ fails to be surjective, there cannot be a single PDE that represents all possible torsions in its root type. Nonetheless, the leaves of dimension at most 4 can be used to fully describe the most symmetric Hessian hydrodynamic PDEs, as in the following theorems.

Theorem 6.2. The root type $\{0\}$ is represented by the wave equation,

$$
u_{22}=u_{13}
$$

and this representation is unique up to $C S p(3)$. Therefore, the wave equation is the unique Hessian hydrodynamic PDE with nine symmetries.

Theorem 6.3. The root type $\{8\}$ is represented by the first flow of the $d K P$ hierarchy,

$$
u_{22}=u_{13}-\frac{1}{2}\left(u_{33}\right)^{2}
$$

and this representation is unique up to $C S p(3)$. Therefore, the first flow of the dKP hierarchy is the unique hydrodynamic PDE with seven symmetries.

Theorem 6.4. The root type $\{7,1\}$ is represented by the PDE

$$
u_{22}=u_{13}-\frac{1}{48}\left(u_{33}\right)^{3}+\frac{1}{2} u_{33} u_{23}
$$

and this representation is unique up to $C S p(3)$. 
Theorem 6.5. The root type $\{6,2\}$ is represented by the PDE

$$
u_{22}=u_{13}+\frac{7\left(u_{23}\right)^{2}}{5 u_{33}-14}
$$

and this representation is unique up to $C S p(3)$.

Theorem 6.6. The root type $\{6,1,1\}$ is represented by the PDE

$$
\begin{aligned}
u_{22}= & u_{13}+\frac{7 u_{23}\left(u_{23}-u_{33}\right)}{\left(5 u_{33}-14\right)}+\frac{49\left(-\left(u_{33}\right)^{2}+14 u_{33}-28\right)}{12\left(5 u_{33}-14\right)} \\
& -\frac{49}{6}\left(\frac{-\left(5 u_{33}-14\right)}{14}\right)^{2 / 5}
\end{aligned}
$$

and this representation is unique up to $C S p(3)$.

Theorems 6.2 through 6.6 are proven via the same technique. For illustration, consider the simplest non-trivial case, Theorem 6.3.

Proof of Theorem 6.3. Consider a 2,3-integrable GL(2)-structure $\pi$ : $\mathcal{B} \rightarrow M$ with $T(b)=x^{8} \frac{1}{322560}$, so $[T(\mathcal{B})]=\{8\}$. The constant is chosen for the aesthetic appeal of the resulting PDE. The goal is to describe Theorem 6.1's embedded hypersurface $i\left(M^{\prime}\right)=\left\{\Pi(h(q)) \in \Lambda^{o}: q\right.$ near $\left.b\right\}$ as the locus of a single equation $F(U)=0$.

As $C S p(3)^{o}$ is a matrix group, the open set $\{g=h(q): q$ near $b\}$ can be described as $\left\{\exp _{I}\left(\eta_{b}(v)\right): v \in \mathbf{T}_{b} \mathcal{B}\right\}$. Moreover, only $v \in \operatorname{ker}(\varphi, \lambda)$ need to be considered, since the fibers of $\pi: \mathcal{B} \rightarrow M$ immerse into the fibers of $\Pi: \operatorname{CSp}(3)^{o} \rightarrow \Lambda^{o}$. Fix an arbitrary $v \in \operatorname{ker}(\varphi, \lambda) \subset \mathbf{T}_{b} \mathcal{B}$ and write $v$ in components using the tautological 1-form, $v\lrcorner \omega=\left(v_{-4}, v_{-2}, v_{0}, v_{2}, v_{4}\right) \in \mathbb{R}^{5}$. Of course, $v\lrcorner \omega$ does not actually provide local coordinates on $\mathcal{B}$ or $M$; however, the matrix $\eta_{b}(v)$ still represents a generic point in $h_{*}\left(\mathbf{T}_{b}(\mathcal{B})\right)$, as seen here:

$$
\eta_{b}(v)=\left(\begin{array}{cccccc}
0 & 0 & 0 & 0 & 0 & 0 \\
0 & 0 & 0 & 0 & 0 & 0 \\
-v_{4} & 0 & 0 & 0 & 0 & 0 \\
v_{-4} & v_{-2} & v_{0} & 0 & 0 & v_{4} \\
v_{-2} & v_{0} & v_{2} & 0 & 0 & 0 \\
v_{0} & v_{2} & v_{4} & 0 & 0 & 0
\end{array}\right)
$$


Therefore,

$$
\exp _{I}\left(\eta_{b}(v)\right)=\left(\begin{array}{cccccc}
1 & 0 & 0 & 0 & 0 & 0 \\
0 & 1 & 0 & 0 & 0 & 0 \\
-v_{4} & 0 & 1 & 0 & 0 & 0 \\
v_{-4}-\frac{1}{6} v_{4}^{3} & v_{-2}+\frac{1}{2} v_{4} v_{2} & v_{0}+\frac{1}{2} v_{4}^{2} & 1 & 0 & v_{4} \\
v_{-2}-\frac{1}{2} v_{4} v_{2} & v_{0} & v_{2} & 0 & 1 & 0 \\
v_{0}-\frac{1}{2} v_{4}^{2} & v_{2} & v_{4} & 0 & 0 & 1
\end{array}\right) .
$$

So, using Equation (1.14), a generic point $U \in i\left(M^{\prime}\right)$ looks like

$$
\begin{aligned}
U & =\Pi\left(\exp _{I}\left(\eta_{p}(v)\right)\right) \\
& =\left(\begin{array}{ccc}
v_{-4}-\frac{1}{6} v_{4}^{3}+\left(v_{0}+\frac{1}{2} v_{4}^{2}\right) v_{4} & v_{-2}+\frac{1}{2} v_{4} v_{2} & v_{0}+\frac{1}{2} v_{4}^{2} \\
v_{-2}+\frac{1}{2} v_{4} v_{2} & v_{0} & v_{2} \\
v_{0}+\frac{1}{2} v_{4}^{2} & v_{2} & v_{4}
\end{array}\right) .
\end{aligned}
$$

There is a single relation between the entries of such $U$ :

$$
U_{22}=U_{13}-\frac{1}{2}\left(U_{33}\right)^{2} .
$$

When $U$ is interpreted as the Hessian of $u: \mathbb{R}^{3} \rightarrow \mathbb{R}$, Equation (6.13) is the first flow of the dKP hierarchy, a well-known example of a Hessian hydrodynamic equation.

The only change for the other root types is that $\eta$ is more complicated; hence, its exponential is (immensely) more difficult to compute, and the relation $F(U)=0$ is more difficult to recognize. Note also that $U=0$ is always in the locus of the equation obtained by this procedure. Therefore, PDEs such as the Boyer-Finley equation, $u_{x x}+u_{y y}=e^{u_{t t}}$ (which has six symmetries and must represent one of the 3-dimensional root types [14]), will not directly appear as representatives via this procedure. None-the-less, every $C S p(3)$ equivalence class of Hessian hydrodynamic PDEs must arise this way.

\subsection{Hyperbolic planar PDEs}

This section presents some preliminary but intriguing observations regarding the hyperbolic linear Pfaffian system $\mathcal{I}$ describing bi-secant surfaces in 
Theorem 3.1 and its relation to hyperbolic second-order planar PDEs,

$$
f\left(\xi^{1}, \xi^{2}, z, z_{1}, z_{2}, z_{11}, z_{12}, z_{22}\right)=0
$$

Equation (6.14) defines a 7-dimensional manifold $\Sigma_{f}=f^{-1}(0) \subset \mathbb{J}^{2}\left(\mathbb{R}^{2}, \mathbb{R}\right)$ whose structure equations are obtained by pulling back the contact system $[18,30]$. Such $\Sigma_{f}$ admit a point-wise classification into Monge-Ampère, Goursat or generic-type equations.

Theorem 6.7. Consider $(\mathcal{B}, M, p)_{2,3}$ with $T(b)=v$ for some $b \in \mathcal{B}_{p}$. Over a neighborhood $M^{\prime}$ of $p$, there is a bundle $W \rightarrow M^{\prime}$ with 7-dimensional fiber and a submersion $f: \mathcal{B}\left(M^{\prime}\right) \rightarrow W$ such that the ideal $\mathcal{I}$ from Theorem 3.1 describing the existence of bi-secant surfaces through $p$ is the pullback of a hyperbolic linear Pfaffian ideal $\overline{\mathcal{I}}$ on $W$. This $W$ admits a coframe $\left(\beta^{1}, \ldots, \beta^{7}\right)$ such that

$$
\begin{aligned}
& d \beta^{1} \equiv \beta^{2} \wedge \beta^{4}+\beta^{3} \wedge \beta^{6}, \quad \bmod \beta^{1}, \\
& d \beta^{2} \equiv U_{1} \beta^{3} \wedge \beta^{7}+\beta^{4} \wedge \beta^{5}, \quad \bmod \beta^{1}, \beta^{2}, \\
& d \beta^{3} \equiv U_{2} \beta^{2} \wedge \beta^{5}+\beta^{6} \wedge \beta^{7}, \quad \bmod \beta^{1}, \beta^{3} .
\end{aligned}
$$

for $U_{1}=-645,120 T_{-8} \frac{\left(\mu_{3}\right)^{3}}{\mu_{2} \mu_{1}} \quad$ and $\quad U_{2}=-645,120 T_{8} \frac{\left(\mu_{2}\right)^{3}}{\mu_{3} \mu_{1}}$ for some nonzero functions $\mu_{1}, \mu_{2}$ and $\mu_{3}$ on $W$.

Moreover, there is some second-order hyperbolic planar PDE $f$ and a diffeomorphism $\varphi: \Sigma_{f} \rightarrow W$ such that these structure equations on $W$ pull back via $\varphi^{*}$ to the contact-induced structure equations on $\Sigma_{f}$.

Proof. Fix $(\mathcal{B}, M, p)_{2,3}$. As in the general case of Theorem 3.1, the linear Pfaffian system describing the existence of bi-secant surfaces through $p$ is differentially generated by $\omega^{-2}, \omega^{0}$ and $\omega^{2}$, and it has tableau given by

$$
d\left(\begin{array}{c}
\omega^{-2} \\
\omega^{0} \\
\omega^{2}
\end{array}\right)=\left(\begin{array}{cc}
\pi_{1} & 0 \\
0 & 0 \\
0 & \pi_{2}
\end{array}\right) \wedge\left(\begin{array}{c}
\omega^{-2} \\
\omega^{4}
\end{array}\right)
$$

for $\pi_{1}=2 \varphi_{2}-322,560 T_{-2} \omega^{4}$ and $\pi_{2}=-2 \varphi_{-2}-322,560 T_{2} \omega^{-4}$. The Lie algebra $A(\mathcal{I})$ of Cauchy characteristics of $\mathcal{I}$ is spanned by the duals of $\lambda$ and $\varphi^{0}$. Therefore, the retracting space $C(\mathcal{I})=A(\mathcal{I})^{\perp}$ is a rank-seven Frobenius system on $\mathcal{B}$, so $\mathcal{B}$ admits a foliation by 2 -dimensional Cauchy characteristic surfaces [3, Section II.2, 22, Section 6.1]. Let $W$ denote the 7-dimensional (local) leaf space for this foliation, so there is a submersion $\tilde{\pi}: \mathcal{B} \rightarrow W$. Since the $\omega$ is semi-basic for the submersion $\tilde{\pi}, W$ also admits a submersion onto 
a neighborhood of $p \in M$. It remains to find the structure equations for a coframing on $W$.

Write $\alpha^{1}=\omega^{0}, \alpha^{2}=\omega^{-2}, \alpha^{3}=\omega^{2}, \alpha^{4}=\pi_{1}, \alpha^{5}=\omega^{-4}, \alpha^{6}=\pi_{2}, \alpha^{7}=$ $\omega^{4}, \alpha^{8}=\varphi_{0}$ and $\alpha^{9}=\lambda$ as a coframe for $\mathcal{B}$. To simplify the notation, fix the index convention $1 \leq i, j, k \leq 7$ and $8 \leq r, s \leq 9$, so the forms $\alpha^{i}$ are semibasic for the bundle $\mathcal{B} \rightarrow W$, and the forms $\alpha^{r}$ are vertical for the bundle $\mathcal{B} \rightarrow W$. Write $d \alpha^{i}=-\frac{1}{2} C_{j k}^{i} \alpha^{j} \wedge \alpha^{k}-C_{j r}^{i} \alpha^{j} \wedge \alpha^{r}$, where $C_{j k}^{i}=-C_{k j}^{i}$ and $C_{j a}^{i}$ are functions of $T$ as determined by Theorem 4.2. Note that $C_{j a}^{i}=0$ if $i \neq j$, and

$$
\begin{aligned}
& C_{18}^{1}=0, \quad C_{28}^{2}=-4, \quad C_{38}^{3}=4, \quad C_{48}^{4}=4, \quad C_{58}^{5}=-8, \\
& C_{68}^{6}=-4, \quad C_{78}^{7}=8, \\
& C_{19}^{1}=1, \quad C_{29}^{2}=1, \quad C_{39}^{3}=1, \quad C_{48}^{4}=0, \quad C_{58}^{5}=1, \\
& C_{68}^{6}=0, \quad C_{78}^{7}=1 .
\end{aligned}
$$

Corollary 2.3 of [3] implies that there exist functions $\mu_{i}$ on $\mathcal{B}$ such that $\frac{1}{\mu_{i}} \alpha^{i}$ is basic. Define a new co-framing $\left(\tilde{\alpha}^{i}\right)$ for $\mathcal{B}$ by setting $\tilde{\alpha}^{i}=\frac{1}{\mu_{i}} \alpha^{i}$ (no sum) and $\tilde{\alpha}^{r}=\alpha^{r}$. Thus, $\mathbf{T}^{*} \mathcal{B}$ is (locally) split into basic 1 -forms and vertical 1-forms with respect to the bundle $\tilde{\pi}: \mathcal{B} \rightarrow W$. Of course, $\tilde{\alpha}^{i}=\tilde{\pi}^{*}\left(\beta^{i}\right)$ for some independent 1 -forms $\beta^{i}$, and $\left(\beta^{1}, \ldots, \beta^{7}\right)$ is the desired co-framing for $W$. Since $\tilde{\alpha}^{i}$ is basic, the structure equations of $\left(\tilde{\alpha}^{i}\right)$ and $\left(\beta^{i}\right)$ are identical.

There is a lot of freedom in the designation of $\mu_{i}$. Write $d \mu_{i}=\mu_{i, j} \tilde{\alpha}^{j}+$ $\mu_{i, r} \tilde{\alpha}^{r}$. The condition that $\tilde{\alpha}^{i}$ is basic is equivalent to $\mu_{i, r}=\mu_{i} C_{i r}^{i}$ for all $1 \leq i \leq 7$ and $8 \leq r \leq 9$, but $\mu_{i, j}$ is otherwise free, so any non-zero function $f_{i}$ on $W$ may be lifted to $\mathcal{B}$ in local coordinates $\left(x^{1}, \ldots, x^{9}\right)$ by setting $\mu_{i}=f_{i} \exp \left(C_{i 8}^{i} x^{8}+C_{i 9}^{i} x^{9}\right)$.

At this point, the coframe $\left(\beta^{i}\right)$ satisfies

$$
\begin{aligned}
d \beta^{1} & \equiv 0, \\
d \beta^{2} & \equiv \frac{\mu_{4} \mu_{5}}{\mu_{2}} \beta^{4} \wedge \beta^{5}, \quad \bmod \left\{\beta^{1}, \beta^{2}, \beta^{3}\right\} \\
d \beta^{3} & \equiv \frac{\mu_{6} \mu_{7}}{\mu_{3}} \beta^{6} \wedge \beta^{7},
\end{aligned}
$$

so one may effectively eliminate $\mu_{5}$ and $\mu_{7}$ by redefining $\beta^{5}$ as $\frac{\mu_{4} \mu_{5}}{\mu_{2}} \beta^{5}$ and $\beta^{7}$ as $\frac{\mu_{6} \mu_{7}}{\mu_{3}} \beta^{7}$. Thus, the structure equations for $W$ satisfy

$$
\begin{aligned}
& d \beta^{1} \equiv 0, \\
& d \beta^{2} \equiv \beta^{4} \wedge \beta^{5}, \quad \bmod \left\{\beta^{1}, \beta^{2}, \beta^{3}\right\} \\
& d \beta^{3} \equiv \beta^{6} \wedge \beta^{7} .
\end{aligned}
$$


One may now re-label the coframe $\left(\beta^{i}\right)$ following the procedure given in $[30$, Appendix A] to obtain Equation (6.15).

Although $U_{1}$ and $U_{2}$ as written in the theorem are not explicitly functions on $W$, this is easily remedied. As noted above, $\mu_{i}$ may be taken as the lift of $f_{i}$ on $W$. Also, the action of $\alpha^{8}$ and $\alpha^{9}$ is diagonal on $T$ :

$$
d\left(\begin{array}{c}
T_{-8} \\
T_{-6} \\
T_{-4} \\
T_{-2} \\
T_{0} \\
T_{2} \\
T_{4} \\
T_{6} \\
T_{8}
\end{array}\right) \equiv\left(\begin{array}{c}
T_{-8} \\
T_{-6} \\
T_{-4} \\
T_{-2} \\
T_{0} \\
T_{2} \\
T_{4} \\
T_{6} \\
T_{8}
\end{array}\right) \alpha^{8}+\left(\begin{array}{c}
16 T_{-8} \\
12 T_{-6} \\
8 T_{-4} \\
4 T_{-2} \\
0 \\
-4 T_{2} \\
-8 T_{4} \\
-12 T_{6} \\
-16 T_{8}
\end{array}\right) \alpha^{9}, \quad \bmod \alpha^{1}, \ldots, \alpha^{7}
$$

So, while there is no natural map from $W$ to $\mathcal{O}_{J}(\mathcal{B})$, the local Lie group $G$ generated by the fiber actions of $H$ and $I_{9}$ (corresponding to $\alpha^{8}$ and $\alpha^{9}$ ) induces a map $W \rightarrow \mathcal{O}_{J}(\mathcal{B}) / G$ that one could also call $T$.

Theorem 11.1.1 of [29] implies that any 7-dimensional manifold with structure equations of this form must be diffeomorphic to $\Sigma_{f}$ for some hyperbolic planar $\operatorname{PDE} f$.

Corollary 6.2. For any Hessian hydrodynamic PDE, the level sets of the Riemann invariants are the solutions of planar hyperbolic PDEs.

Proof. The manifold $W$ arises from the ideal describing bi-secant surfaces in $M$, and Corollary 4.1 shows that these are the level sets of the Riemann invariants of the Hessian hydrodynamic PDE defining $\mathcal{B}$.

This corollary is not at all surprising, since the entire point of hydrodynamic reduction is to reduce a hyperbolic PDE in three variables to a family of hyperbolic planar PDEs defined by the Riemann invariants.

In the case of 2,3-integrability, Corollary 6.2 also provides a more explicit justification for the claim in Theorem 3.1 that the PDE defining bi-secant surfaces can be solved in the smooth category with smooth initial data. One naturally asks "to which $\Sigma_{f}$ is $W$ equivalent?"

Corollary 6.3. Let $\Sigma_{f}=f^{-1}(0) \subset \mathbb{J}^{2}\left(\mathbb{R}^{2}, \mathbb{R}\right)$ for a hyperbolic planar PDE $f$. Suppose there is a local diffeomorphism $\psi: \Sigma_{f} \rightarrow W$ with $\tilde{\pi}(b) \in \psi\left(\Sigma_{f}\right)$. 
(1) If $x=0$ and $y=0$ are both roots of $T(b)$, then $\Sigma_{f}$ is of the MongeAmpère type at $\psi^{-1}(\tilde{\pi}(b))$.

(2) If exactly one of $x=0$ or $y=0$ is a root of $T(b)$, then $\Sigma_{f}$ is of the Goursat type at $\psi^{-1}(\tilde{\pi}(b))$.

(3) If neither $x=0$ nor $y=0$ is a root of $T(b)$, then $\Sigma_{f}$ is of the generic type at $\psi^{-1}(\tilde{\pi}(b))$.

Proof. These are the three pointwise types of planar hyperbolic PDEs, and for structure equations of the form in Equation (6.15), they are determined by whether $U_{1}$ and $U_{2}$ vanish $[18,30]$. Since $U_{1} \sim T_{-8}, U_{1}=0$ if and only if $y=0$ is a root of $T(b) \in \mathcal{V}_{8}$. Similarly, $U_{2}=0$ if and only if $x=0$ is a root of $T(b) \in \mathcal{V}_{8}$.

Corollary 6.3 puts interesting restrictions on which GL(2)-structures can yield which planar PDEs. In particular, $x=0$ and $y=0$ can both be roots of $v=T(b)$ if and only if $[v]$ is a root type having two distinct real roots, and the strictly complex root types cannot have $x=0$ or $y=0$ as roots. Notably, the type of $W$ can change. For example, suppose $T(b)=x^{7} y$, so $\psi^{-1}(\tilde{\pi}(b))$ is of the Monge-Ampère type but nearby $\psi^{-1}\left(\tilde{\pi}\left(b^{\prime}\right)\right)$ is of the generic type, since $T\left(b^{\prime}\right)=\left(x+\varepsilon_{1} y\right)^{7}\left(\varepsilon_{2} x+y\right)$. This type-changing does not occur for the flat structure, and it is easy to compute a change-of-frame from the structure equations of the flat $W$ to the structure equations of $\Sigma_{f}$ for the planar wave equation $z_{12}=0$.

Corollary 6.4. Let $(\mathcal{B}, M, p)_{2,3}$ be flat. Then $W$ is isomorphic to $\left\{z_{12}=\right.$ $0\} \subset \mathbb{J}^{2}\left(\mathbb{R}^{2}, \mathbb{R}\right)$.

\section{Concluding remarks}

The main results of this article are summarized in figure 2. In short, Hessian hydrodynamic PDEs in three independent variables are equivalent to local 2,3-integrable GL(2)-structures of degree 4, and both objects admit a geometric, coordinate-free classification by the singular foliation of $\mathbb{R}^{9}$ shown in the figure.

Lemma 5.1 seems to be a miraculous coincidence. The bluntness of this relationship between $v$ and $J(v)$ prompted me to investigate relationships between the roots of $v$ and the structure of the leaf $\mathcal{O}_{J}(v)$, yielding this project's main result, Theorem 5.1. It appears that no such relationship holds for 2,3-integrable GL(2)-structures of degree $n \geq 5$, even though versions of Theorem 4.2 and Lemma 4.3 exist in those cases. In general, 


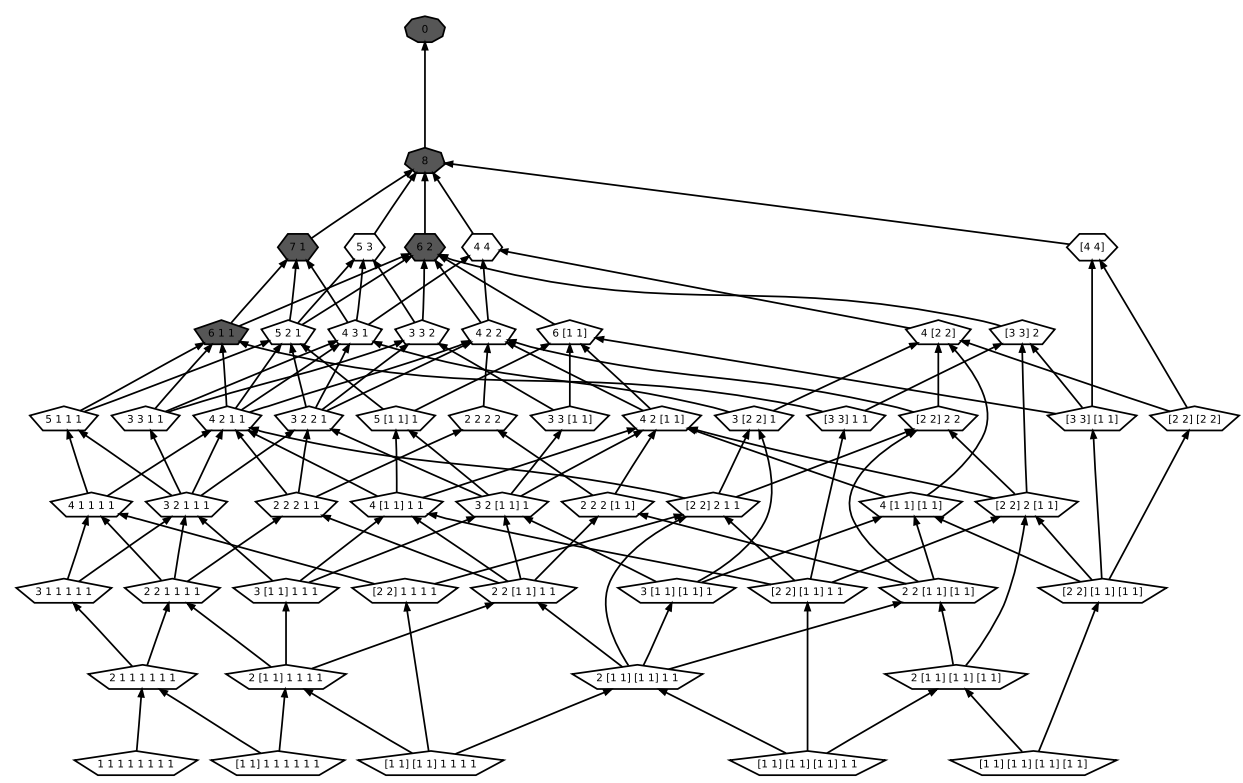

Figure 2: The leaf-classification of all $(\mathcal{B}, M, p)_{2,3}$. The number of sides on a node is the dimension of the bundle after the symmetry reduction from Section 5.1. Representative PDEs for the shaded nodes are included in Section 6.

intransitive groupoids and pseudo-groups are very poorly understood, and it is generally impossible to explicitly write down the integral manifolds of any given singular distribution. If it were not for the coincidence in this case, the leaf-equivalence classes would have little utility in understanding the Hessian hydrodynamic PDEs.

Of course, Hessian hydrodynamic PDEs on $u: \mathbb{R}^{3} \rightarrow \mathbb{R}$ are not the only integrable PDEs of interest in mathematics and physics. Two generalizations are important to consider:

Q1. Integrability should be a contact-invariant property of a PDE. The Hessian-only form $F\left(u_{11}, u_{12}, u_{13}, u_{22}, u_{23}, u_{33}\right)=0$ is not preserved under the full family of contact transformations. However, the associated GL(2)-structures apparently relied on this form and its associated $C S p(3)$ transformations. How can this classification be extended to second-order PDEs in three independent variables that also include lower derivatives? How does the GL(2) geometry generalize to these PDEs? The observations and computations of some recent articles may prove very useful $[7,15]$. 
Q2. Very few integrable PDEs are known to exist in more than three independent variables, but equations of the form $F\left(u_{11}, \ldots, u_{N N}\right)=0$ can sometimes yield distributions of rational normal cones of degree $n$ on hypersurfaces $M^{n}=F^{-1}(0) \subset \operatorname{Sym}^{2}\left(\mathbb{R}^{N}\right)$ with $n=\frac{1}{2} N(N+1)-2$. Results similar to those in Section 4 are known for 2,3-integrability for degrees 5 through 20, but the foliation by groupoid orbits of $\mathcal{V}_{n+4}$ is not understood, and $k$-integrability is extremely restrictive [27]. What can integrable GL(2) geometry say about the existence of integrable PDEs in more variables?

On a more detailed level, it would be interesting to study the foliation that appears in the present case. Despite the significant computational difficulties, it is important both to produce representative PDEs for each root type and to find the root types of the well-known Hessian hydrodynamic PDEs. For example, if one can produce Hessian hydrodynamic PDEs as well as contact-equivalent PDEs that involve lower-order terms, such computations could open the door to Q1, above. Additionally, the lack of surjectivity of $T: \mathcal{B} \rightarrow \mathcal{O}_{J}(\mathcal{B})$ is irritating. What is the exact relationship between two PDEs that are leaf-equivalent but do not have overlapping torsion?

Finally, the relationship between $W$, which describes bi-secant surfaces in $M$, and $\Sigma_{f}$, which arises from a planar hyperbolic PDE, is worth pursuing. What is the nature of the correspondence? Can every hyperbolic planar PDE appear this way? Can this correspondence provide any new information about the Riemann invariants of the Hessian hydrodynamic PDE?

\section{Appendix A: The matrix $J(T)$}

For reference, here is the matrix $J(T)$, listed by column. Note! in the $\omega$ columns, the common factor of $9216=2^{10} 3^{2}$ has been removed for clarity.

The $9216 \omega^{-4}$ column:

$$
\left(\begin{array}{c}
280 T_{-8} T_{4}-280 T_{-6} T_{2} \\
-245 T_{-4} T_{2}+70 T_{-8} T_{6}+175 T_{-6} T_{4} \\
70 T_{-4} T_{4}-210 T_{-2} T_{2}+130 T_{-6} T_{6}+10 T_{-8} T_{8} \\
-175 T_{0} T_{2}-35 T_{-2} T_{4}+35 T_{-6} T_{8}+175 T_{-4} T_{6} \\
84 T_{-4} T_{8}+196 T_{-2} T_{6}-140 T_{2}^{2}-140 T_{0} T_{4} \\
-350 T_{2} T_{4}+175 T_{0} T_{6}+175 T_{-2} T_{8} \\
350 T_{0} T_{8}-350 T_{4}^{2} \\
700 T_{2} T_{8}-700 T_{6} T_{4} \\
-1400 T_{6}^{2}+1400 T_{8} T_{4}
\end{array}\right)
$$


The $9216 \omega^{-2}$ column:

$$
\left(\begin{array}{c}
-1400 T_{-8} T_{2}+1400 T_{-6} T_{0} \\
-385 T_{-8} T_{4}-840 T_{-6} T_{2}+1225 T_{-4} T_{0} \\
-280 T_{-4} T_{2}+1050 T_{-2} T_{0}-70 T_{-8} T_{6}-700 T_{-6} T_{4} \\
-910 T_{-4} T_{4}+280 T_{-2} T_{2}+875 T_{0}^{2}-240 T_{-6} T_{6}-5 T_{-8} T_{8} \\
1540 T_{0} T_{2}-952 T_{-2} T_{4}-28 T_{-6} T_{8}-560 T_{-4} T_{6} \\
-105 T_{-4} T_{8}-1120 T_{-2} T_{6}+1400 T_{2}^{2}-175 T_{0} T_{4} \\
2100 T_{2} T_{4}-1750 T_{0} T_{6}-350 T_{-2} T_{8} \\
2450 T_{4}^{2}-1050 T_{0} T_{8}-1400 T_{2} T_{6} \\
-2800 T_{2} T_{8}+2800 T_{6} T_{4}
\end{array}\right)
$$

The $9216 \omega^{0}$ column:

$$
\left(\begin{array}{c}
-2800 T_{-6} T_{-2}+2800 T_{-8} T_{0} \\
-2450 T_{-4} T_{-2}+875 T_{-8} T_{2}+1575 T_{-6} T_{0} \\
210 T_{-8} T_{4}-2100 T_{-2}^{2}+1540 T_{-6} T_{2}+350 T_{-4} T_{0} \\
1890 T_{-4} T_{2}-2625 T_{-2} T_{0}+35 T_{-8} T_{6}+700 T_{-6} T_{4} \\
1568 T_{-4} T_{4}+336 T_{-2} T_{2}-2100 T_{0}^{2}+192 T_{-6} T_{6}+4 T_{-8} T_{8} \\
-2625 T_{0} T_{2}+1890 T_{-2} T_{4}+35 T_{-6} T_{8}+700 T_{-4} T_{6} \\
210 T_{-4} T_{8}+1540 T_{-2} T_{6}-2100 T_{2}^{2}+350 T_{0} T_{4} \\
-2450 T_{2} T_{4}+1575 T_{0} T_{6}+875 T_{-2} T_{8} \\
2800 T_{0} T_{8}-2800 T_{2} T_{6}
\end{array}\right)
$$

The $9216 \omega^{2}$ column:

$$
\left(\begin{array}{c}
2800 T_{-4} T_{-6}-2800 T_{-2} T_{-8} \\
-1400 T_{-6} T_{-2}-1050 T_{-8} T_{0}+2450 T_{-4}^{2} \\
2100 T_{-4} T_{-2}-350 T_{-8} T_{2}-1750 T_{-6} T_{0} \\
-105 T_{-8} T_{4}+1400 T_{-2}^{2}-1120 T_{-6} T_{2}-175 T_{-4} T_{0} \\
-952 T_{-4} T_{2}+1540 T_{-2} T_{0}-28 T_{-8} T_{6}-560 T_{-6} T_{4} \\
-910 T_{-4} T_{4}+280 T_{-2} T_{2}+875 T_{0}^{2}-240 T_{-6} T_{6}-5 T_{-8} T_{8} \\
1050 T_{0} T_{2}-280 T_{-2} T_{4}-70 T_{-6} T_{8}-700 T_{-4} T_{6} \\
1225 T_{0} T_{4}-385 T_{-4} T_{8}-840 T_{-2} T_{6} \\
1400 T_{0} T_{6}-1400 T_{-2} T_{8}
\end{array}\right)
$$


The $9216 \omega^{4}$ column:

$$
\left(\begin{array}{c}
1400 T_{-4} T_{-8}-1400 T_{-6}^{2} \\
-700 T_{-4} T_{-6}+700 T_{-2} T_{-8} \\
-350 T_{-4}^{2}+350 T_{-8} T_{0} \\
-350 T_{-4} T_{-2}+175 T_{-8} T_{2}+175 T_{-6} T_{0} \\
84 T_{-8} T_{4}-140 T_{-2}^{2}+196 T_{-6} T_{2}-140 T_{-4} T_{0} \\
-35 T_{-4} T_{2}-175 T_{-2} T_{0}+35 T_{-8} T_{6}+175 T_{-6} T_{4} \\
70 T_{-4} T_{4}-210 T_{-2} T_{2}+130 T_{-6} T_{6}+10 T_{-8} T_{8} \\
70 T_{-6} T_{8}-245 T_{-2} T_{4}+175 T_{-4} T_{6} \\
-280 T_{-2} T_{6}+280 T_{-4} T_{8}
\end{array}\right)
$$

The $\lambda, \varphi_{-2}, \varphi_{0}$ and $\varphi_{2}$ columns:

$$
\left(\begin{array}{c}
T_{-8} \\
T_{-6} \\
T_{-4} \\
T_{-2} \\
T_{0} \\
T_{2} \\
T_{4} \\
T_{6} \\
T_{8}
\end{array}\right), \quad\left(\begin{array}{c}
-16 T_{-6} \\
-14 T_{-4} \\
-12 T_{-2} \\
-10 T_{0} \\
-8 T_{2} \\
-6 T_{4} \\
-4 T_{6} \\
-2 T_{8} \\
0
\end{array}\right), \quad\left(\begin{array}{c}
16 T_{-8} \\
12 T_{-6} \\
8 T_{-4} \\
4 T_{-2} \\
0 \\
-4 T_{2} \\
-8 T_{4} \\
-12 T_{6} \\
-16 T_{8}
\end{array}\right), \quad\left(\begin{array}{c}
0 \\
2 T_{-8} \\
4 T_{-6} \\
6 T_{-4} \\
8 T_{-2} \\
10 T_{0} \\
12 T_{2} \\
14 T_{4} \\
16 T_{6}
\end{array}\right) .
$$

\section{Acknowledgments}

I owe many thanks to Robert Bryant and Niky Kamran for their wisdom, guidance, and support, to Dennis The and Eugene Ferapontov for their many helpful comments, and to Jeanne Clelland, whose "Cartan" package for MAPLE made the computations bearable.

This work is supported by an NSF All-Institutes Postdoctoral Fellowship administered by the Mathematical Sciences Research Institute through its core grant DMS-0441170. The author is hosted by the Department of Mathematics and Statistics at McGill University.

\section{References}

[1] L.V. Ahlfors, Complex analysis, 3rd ed., McGraw-Hill Book Co., New York, 1978; An introduction to the theory of analytic functions of one complex variable, International Series in Pure and Applied Mathematics. 
[2] I. Berchenko and P.J. Olver, Symmetries of polynomials, J. Symbolic Comput. 29 (4-5) (2000), 485-514; Symbolic computation in algebra, analysis, and geometry (Berkeley, CA, 1998).

[3] R.L. Bryant, S.S. Chern, R.B. Gardner, H.L. Goldschmidt and P.A. Griffiths, Exterior differential systems, Mathematical Sciences Research Institute Publications, 18, Springer-Verlag, New York, 1991.

[4] R. Bryant, P. Griffiths and D. Grossman, Exterior differential systems and Euler-Lagrange partial differential equations, Chicago Lectures in Mathematics, University of Chicago Press, Chicago, IL, 2003.

[5] R.L. Bryant, Two exotic holonomies in dimension four, path geometries, and twistor theory, in 'Complex geometry and Lie theory (Sundance, UT, 1989)', Proc. Sympos. Pure Math., 53, Amer. Math. Soc., Providence, RI, 1991, 33-88.

[6] R.L. Bryant, Bochner-Kähler metrics, J. Amer. Math. Soc. 14 (3) (2001), 623-715 (electronic).

[7] P.A. Burovskiy, E.V. Ferapontov and S.P. Tsarev, Second order quasilinear pdes and conformal structures in projective space, Int. J. Math. 21 (6) (2010), 779-841, arXiv:0802.2626v3 [nlin.SI].

[8] É. Cartan, Sur la structure des groupes infinis de transformation, Ann. Sci. École Norm. Sup. (3) 21 (1904), 153-206.

[9] E. Cartan, Les groupes de transformations continus, infinis, simples, Ann. Sci. École Norm. Sup. (3) 26 (1909), 93-161.

[10] K.C. Chu, On the geometry of the moduli space of real binary octics, arXiv:0708.0419v1 [math.AG] (2007).

[11] J.B. Conway, Functions of one complex variable, 2nd ed., Graduate Texts in Mathematics, 11, Springer-Verlag, New York, 1978.

[12] M. Crainic and R.L. Fernandes, Integrability of Lie brackets, Ann. Math. (2) 157 (2) (2003), 575-620.

[13] P. Crooks and R. Milson, On projective equivalence of univariate polynomial subspaces, SIGMA Symmetry Integrability Geom. Methods Appl. 5 (107) (2009), 24p. doi:10.3842/SIGMA.2009.107 arXiv:0902.1106v7 [math.QA]. 
[14] E.V. Ferapontov, L. Hadjikos and K.R. Khusnutdinova, Integrable equations of the dispersionless hirota type and hypersurfaces in the lagrangian grassmannian, International Mathematics Research Notices (2009), doi:10.1093/imrn/rnp134 arXiv:0705.1774v1 [nlin.SI].

[15] E.V. Ferapontov and K.R. Khusnutdinova, Hydrodynamic reductions of multi-dimensional dispersionless PDEs: the test for integrability, J. Math. Phys. 4(6) (2004), 2365-2377.

[16] E.V. Ferapontov and K.R. Khusnutdinova, On the integrability of $(2+$ 1)-dimensional quasilinear systems, Comm. Math. Phys. 248(1) (2004), 187-206.

[17] E.V. Ferapontov, D.A. Korotkin and V.A. Shramchenko, Boyer-Finley equation and systems of hydrodynamic type, Classical Quantum Gravity 19(24) (2002), L205-L210.

[18] R.B. Gardner and N. Kamran, Characteristics and the geometry of hyperbolic equations in the plane, J. Differential Equations 104(1) (1993), 60-116.

[19] R.B. Gardner, The method of equivalence and its applications, CBMSNSF Regional Conference Series in Applied Mathematics, 58, Society for Industrial and Applied Mathematics (SIAM), Philadelphia, PA, 1989.

[20] J. Harris, Algebraic geometry, Graduate Texts in Mathematics, 133, Springer-Verlag, New York, 1995, A first course, Corrected reprint of the 1992 original.

[21] J.E. Humphreys, Introduction to Lie algebras and representation theory, xii+169, Graduate Texts in Mathematics, 9. Springer, New York, 1972.

[22] T.A. Ivey and J.M. Landsberg, Cartan for beginners: differential geometry via moving frames and exterior differential systems, Graduate Studies in Mathematics, 61, American Mathematical Society, Providence, RI, 2003.

[23] K.C.H. Mackenzie, General theory of Lie groupoids and Lie algebroids, London Mathematical Society Lecture Note Series, 213, Cambridge University Press, Cambridge, 2005.

[24] P. Nurowski, Comment on GL(2,R) geometry of 4 th order ODEs, arXiv:0710.1658v1 [math.DG] (2007). 
[25] P.J. Olver, Equivalence, invariants, and symmetry, Cambridge University Press, Cambridge, 1995.

[26] A.D. Smith, Maple code for integrable GL(2)-structures, WWW, 10 2009, http://www.math.mcgill.ca/adsmith/GL2structures.mpl.

[27] A.D. Smith, Integrability of second-order partial differential equations and the geometry of GL(2)-structures, $\mathrm{PhD}$ thesis, Duke University, North Carolina, United States, April 2009.

[28] P. Stefan, Integrability of systems of vector fields, J. London Math. Soc. (2) $21(3)$ (1980), 544-556.

[29] O. Stormark, Lie's structural approach to PDE systems, Encyclopedia of Mathematics and its Applications, 80, Cambridge University Press, Cambridge, 2000.

[30] D. The, Contact geometry of hyperbolic equations of generic type, SIGMA Symmetry Integrability Geom. Methods Appl. 4 (2008), Paper 058,52 .

[31] S.P. Tsarëv, The geometry of Hamiltonian systems of hydrodynamic type. The generalized hodograph method, Izv. Akad. Nauk SSSR Ser. Mat. 54(5) (1990), 1048-1068.

[32] S.P. Tsarëv, Classical differential geometry and integrability of systems of hydrodynamic type, Applications of analytic and geometric methods to nonlinear differential equations (Exeter, 1992), NATO Adv. Sci. Inst. Ser. C Math. Phys. Sci., 413, Kluwer Acad. Publ., Dordrecht, 1993, 241-249.

[33] S.P. Tsarëv, Integrability of equations of hydrodynamic type from the end of the 19th to the end of the 20th century, Integrability: the Seiberg-Witten and Whitham equations (Edinburgh, 1998), Gordon and Breach, Amsterdam, 2000, 251-265.

[34] Deane Yang, Involutive hyperbolic differential systems, Mem. Amer. Math. Soc. 68(370) (1987), xii+93.

[35] V.E. Zakharov (ed.), What is integrability?, Springer Series in Nonlinear Dynamics, Springer-Verlag, Berlin, 1991.

[36] D. Zwillinger, S.G. Krantz and K.H. Rosen (eds.), CRC standard mathematical tables and formulae, 30th ed., CRC Press, Boca Raton, FL, 1996. 
Department of Mathematics and Statistics

MCGILl UNIVERSITY

Montreal, Quebec H3A 2K6

CANADA

E-mail address: adsmith@msri.org

ReCeIVEd JANuARY 13, 2010 